\title{
V838 Monocerotis as seen by ALMA: A remnant of a binary merger in a triple system ${ }^{\star}$
}

\author{
Tomek Kamiński ${ }^{1} \oplus$, Romuald Tylenda ${ }^{1}$, Aleksandra Kiljan², Mirek Schmidt ${ }^{1}$, Krzysztof Lisiecki $^{3}$, Carl Melis ${ }^{4}$, \\ Adam Frankowski ${ }^{1}$, Vishal Joshi ${ }^{5}$, and Karl M. Menten ${ }^{6}$ \\ ${ }^{1}$ Nicolaus Copernicus Astronomical Center, Polish Academy of Sciences, Rabiańska 8, 87-100 Toruń, Poland \\ e-mail: tomkam@ncac.torun.pl \\ 2 Warsaw University Astronomical Observatory, Al. Ujazdowskie 4, 00-478 Warszawa, Poland \\ ${ }^{3}$ Institute of Astronomy, Nicolaus Copernicus University in Toruń, Grudziądzka 5, 87-100 Toruń, Poland \\ ${ }^{4}$ Center for Astrophysics and Space Sciences, University of California, San Diego, CA 92093-0424, USA \\ ${ }^{5}$ Physical Research Laboratory, Navrangpura, Ahmedabad, Gujarat 380009, India \\ ${ }^{6}$ Max-Planck-Institut für Radioastronomie, Auf dem Hügel 69, 53-121 Bonn, Germany
}

Received 11 June 2021 / Accepted 4 September 2021

\begin{abstract}
Context. V838 Mon erupted in 2002, quickly becoming the prototype of a new type of stellar eruption known today as the (luminous) red nova. Red nova outbursts are thought to be caused by stellar mergers. The merger in V838 Mon took place in a triple or higher system involving two B-type stars.

Aims. We wish to characterize the merger remnant $\sim 17 \mathrm{yr}$ after the eruption to learn more about the remaining system, the progenitor, and the merger physics.

Methods. We mapped the merger site with ALMA at a resolution of $\sim 25$ mas, or 148 au for a distance of $5.9 \mathrm{kpc}$, in continuum dust emission and in rotational lines of simple molecules, including $\mathrm{CO}, \mathrm{SiO}, \mathrm{SO}, \mathrm{SO}_{2}, \mathrm{AlOH}$, and $\mathrm{H}_{2} \mathrm{~S}$. We use radiative transfer calculations to reproduce the architecture of the remnant at the epoch of the ALMA observations. We also make use of optical spectroscopy data obtained mainly with VLT/X-shooter and SALT/HRS.

Results. For the first time, we identify the position of the B-type companion relative to the outbursting component of V838 Mon. The stellar remnant is surrounded by a clumpy wind with characteristics similar to those of the winds of red supergiants. The merger product is also associated with an elongated structure of $17.6 \times 7.6$ mas $(104 \times 45 \mathrm{au})$ seen in continuum emission, and which we interpret as a disk seen at a moderate inclination. Maps of continuum and molecular emission also show a complex region of interaction between the B-type star (its gravity, radiation, and wind) and the flow of matter ejected in 2002 . The remnant's molecular mass is about $0.1 M_{\odot}$ and the dust mass is $8.3 \times 10^{-3} M_{\odot}$. The mass of the atomic component remains unconstrained.

Conclusions. The most interesting region for understanding the merger of V838 Mon lies in its direct vicinity and appears elongated, but details of its substructure remain unknown. To study it further and in more detail will require even higher angular resolutions. ALMA maps show us an extreme form of interaction between the merger ejecta and the distant ( $250 \mathrm{au})$ companion. This interaction is similar to that known from the Anteres AB system but at a much higher mass loss rate. The B-type star not only deflects the merger ejecta but also changes its molecular composition with an involvement of circumstellar shocks. The ALMA view of V838 Mon offers the best images of a merger site so far.
\end{abstract}

Key words. astrochemistry - shock waves - stars: individual: V838 Mon - circumstellar matter - techniques: interferometric

\section{Introduction}

V838 Monocerotis (V838 Mon) underwent a red nova event in early 2002 (Munari et al. 2002b; Kimeswenger et al. 2002; Crause et al. 2003; Tylenda et al. 2005; Tylenda 2005, and references therein). Its spectacular outburst to a luminosity of $10^{6} L_{\odot}$ (Sparks et al. 2008; Tylenda 2005) was associated with an iconic light echo (Bond et al. 2003; Tylenda 2004; Tylenda \& Kamiński 2012), reflected in a near interstellar cloud (Kamiński et al. 2011). This drew a lot of attention to V838 Mon, even among nonastronomers. The leading hypothesis explaining its eruption is a merger between two young $(<25$ Myr; Kamiński 2008; Afşar \& Bond 2007) stars (Tylenda \& Soker 2006) of masses of approximately 8.0 and $0.4 M_{\odot}$ (Tylenda et al. 2005). Spectra obtained during the eruption showed that the 2002 event produced ejecta with velocities approaching $500 \mathrm{~km} \mathrm{~s}^{-1}$ (e.g.,

\footnotetext{
* Movies associated with Figs. 9 and 12 are available at https://www . aanda.org
}

Wisniewski et al. 2003). After three months of high but varying brightness, the object entered a phase of decline which, characteristically for red novae, resulted in a very cool remnant with a photospheric temperature possibly as low as $2000-2300 \mathrm{~K}$ (Evans et al. 2003; Pavlenko et al. 2006). Since then, the circumstellar material has become primarily apparent through atomic lines and molecular bands characterized by a very low excitation temperature of typically a few hundred Kelvin (Kamiński et al. 2009). In the cool post-outburst phase in late 2002, the merger ejecta started forming dust (Tylenda 2005; Wisniewski et al. 2008). Here, we present observations of the cool circumstellar remnant about $17 \mathrm{yr}$ after the eruption. With millimeter ( $\mathrm{mm}$ ) observations at 20 mas resolution, we spatially resolve both gas and dust in the circumstellar environment for the first time.

In early observations of the outburst, it was recognized that the erupting object lies close to a B-type main-sequence star (Wagner \& Starrfield 2002; Munari et al. 2002a). Followup observations revealed that it is a physical companion to 
V838 Mon (Tylenda et al. 2009) and that with a few other Btype stars it belongs to a scant open cluster (Afşar \& Bond 2007; Ortiz-León et al. 2020) in the far outer Galaxy, at a distance of $5.9 \pm 0.4 \mathrm{kpc}$ (Sparks et al. 2008; Ortiz-León et al. 2020). Pre-outburst photometry suggests that V838 Mon was an early B-type star as well (Tylenda et al. 2005). The presence of the companion makes the merger scenario of V838 Mon particularly interesting as it happened in a triple (or a higher multiple) system.

Not much is known about the B-type companion. A few of its spectral features, always entangled with the much brighter spectrum of the luminous red remnant of the merger, revealed its spectral type of B3 V (or an effective temperature of $18000 \mathrm{~K}$ ) and a fast rotation rate of $v \sin i=250 \pm 50 \mathrm{~km} \mathrm{~s}^{-1}$ (Kamiński et al. 2009). However, V838 Mon appears to be under-luminous compared to the other B-type stars in the cluster, a problem which has not yet been explained (Tylenda \& Kamiński 2012). In 2005, the signatures of the B star disappeared. This was first interpreted as an eclipse (Goranskij 2006; Munari et al. 2007), but further studies showed that the blue star vanished because it became completely embedded in the dusty ejecta produced in the 2002 eruption (Tylenda et al. 2009). Prior to the complete disappearance from the optical, the B star started photo-ionizing the approaching ejecta, producing an emission spectrum that included relatively strong lines of [Fe II] (Munari et al. 2007; Kamiński et al. 2009). Based on these features and the timing of the "eclipse", Tylenda et al. (2009) found that the separation between V838 Mon and the B star is about $250 \mathrm{au}$. This latter calculation relied on the gas velocity, which was not straightforward to infer given that the ejecta velocities varied considerably over the 2002 event, and it was uncertain whether spherical symmetry could be assumed for the merger remnant. Here we present observations which for the first time directly reveal the location of the B star with respect to V838 Mon.

Currently, the stellar remnant of V838 Mon is a luminous and cool star with a wind-like outflow (Tylenda et al. 2009) and active but decaying $\mathrm{SiO}$ masers (Deguchi et al. 2005; Ortiz-León et al. 2020) which are commonly associated with outflows from masslosing asymptotic giant branch (AGB) stars and red supergiants. V838 Mon shares a lot more of its observational characteristics with red supergiants. As an M type "supergiant" with a main-sequence B-type companion, the system can be technically classified as being of the rare VV Ceph type (Neugent et al. 2019; Cowley 1969). Here, we demonstrate more specifically that it can be treated as an analog of the binary system of Antares, but with a much denser outflow and much more pronounced interaction effects between the cool dusty wind and the hot star.

The present paper is organized as follows. In Sect. 2, we present technical details of the high-resolution observations of V838 Mon with the Atacama Large Millimeter/submillimeter Array (ALMA). The subsequent two sections present results of these observations in continuum (Sect. 3) and in line emission (Sect. 4). Section 5 briefly introduces optical observations of the remnant before and after the ALMA epoch to indicate the status of V838 Mon in the context of the temporal evolution since the end of the outburst. In Sect. 6, we present our threedimensional (3D) reconstruction of the remnant, primarily based on the dust emission data. We discuss all of our results in Sect. 7 and summarize them in Sect. 8.

\section{ALMA observations}

The ALMA observations were acquired on 20 June 2019 in three consecutive execution blocks. The array with 43 antennas was in its most extended configuration with baselines of 83.1$15238.4 \mathrm{~m}$. Visibilities were calibrated with default calibration scripts delivered by the observatory and data were processed, including imaging, with the Common Astronomy Software Applications (CASA; McMullin et al. 2007). An attempt to improve data quality by a self-calibration procedure was unsatisfactory, and visibilities with the original calibration were used. The observations were executed in band 6 and covered frequencies 215.56-217.44, 219.06-220.94, 230.11-231.99, and 232.06-233.94 GHz at a native resolution of $1.3-2.5 \mathrm{~km} \mathrm{~s}^{-1}$. These spectral ranges overlap with older interferometric observations of V838 Mon with the Submillimeter Array (SMA), which are nevertheless of lower angular resolution and much poorer sensitivity (Kamiński et al. 2018). The continuum sensitivity of the ALMA data is $9.8 \mu \mathrm{Jy}$ per beam at a beam full width at half maximum (FWHM) of $28 \times 25$ mas and with natural weighting of visibilities. Continuum images include only line-free channels and cubes with line emission had continuum removed by a linear (zeroth-order) fit to calibrated visibilities. Our absolute astrometric accuracy measured as a displacement of a check source from its ALMA catalog position was 18 mas. Parts of the ALMA dataset were briefly presented in Ortiz-León et al. (2020).

\section{ALMA results: continuum}

We first present results for the continuum emission imaged at an effective frequency of $224.75 \mathrm{GHz}$ or $1.334 \mathrm{~mm}$. For the analysis, images were produced at different weighting schemes. An example map with Briggs weighting and the robust parameter of $R=0.5$ (resulting in a beam of a FWHM of 22 mas) is shown in Fig. 1. There are three main emission components seen in the continuum maps. The strongest one is centered at International Celestial Reference System (ICRS) J2000.0 coordinates $\mathrm{RA}=07: 04: 04.821535( \pm 0.000012)$ and Dec $=-03: 50: 50.629426( \pm 0.000177)$. The positional uncertainties here reflect only the fit errors and do not account for uncertainties in the astrometric calibration. Within the errors, the location is consistent with a VLBI position of the masers of V838 Mon (Ortiz-León et al. 2020). The continuum source can be identified as emission of circumstellar material in the direct vicinity of the stellar remnant of the 2002 eruption. We hereafter refer to this source as "component M". A Gaussian fit to this compact source (at Briggs' $R=0.5$ ) gives a beam-deconvolved size of $(17.6 \pm 0.8) \times(7.6 \pm 1.4)$ mas with the major axis at a position angle (PA) of $132^{\circ} \pm 4^{\circ}$. Its flux of $588 \pm 14 \mu \mathrm{Jy}$ constitutes $28 \%$ of the total continuum flux in the map. The position angle of the mm component is close to $\mathrm{PA}=170^{\circ} \pm 30^{\circ}$ of an elongated mid-infrared (MIR) structure surrounding V838 Mon and found in Very Large Telescope Interferometer (VLTI) observations by Chesneau et al. (2014) in 2011 and by Mobeen et al. (in prep.) in 2019.

The secondary component is centered at ICRS coordinates RA =07:04:04.819359 $( \pm 0.000051)$ and Dec $=-$ 03:50:50.609454 ( \pm 0.000965$)$, or 32.6 mas west and 20.0 mas north (38.2 mas at a PA of -58.5) from the main component. A Gaussian fit yields a beam-deconvolved size of $(24.0 \pm 3.5) \times(18.5 \pm 3.5)$ mas (i.e., almost circular) at a PA of $166^{\circ} \pm 45^{\circ}$ and a flux of $510 \pm 48 \mu \mathrm{Jy}(24.4 \%$ of the total flux in the map). We identify the component as material directly surrounding the B-type companion of V838 Mon and hereafter refer to it as the "B component". Its projected distance to V838 Mon of $225 \mathrm{au}$ (at the $5.9 \mathrm{kpc}$ distance) is close to the separation $a \approx 250$ au calculated from the timing of the interaction of the B-type star with the merger ejecta (Tylenda et al. 2009). 
This close match between the physical and projected distances suggests that the binary orbit is almost in the sky plane. There is no millimeter emission bridging the two stars, that is, the two Gaussian components account for all emission seen around the stars at our sensitivity level.

The third component is much more extended and contains all the remaining emission (47.6\% of the total or $1.0 \mathrm{mJy}$ ). It has an irregular shape and an approximate size of $135 \times 70$ mas. Its outer parts have low surface brightness and maps at a better sensitivity may reveal that it is even more extended. Hereafter, we refer to this component as "the extended cloud". Its peak emission is located at $\approx 100$ mas or 590 au from the V838 Mon position. A line connecting the peak cloud emission with that of V838 Mon does not directly cross the position of the B component, but there are cloud parts directly on the line connecting $M$ and B (see the residual map in Fig. 1). Some weak diffuse emission is seen north and southwest of and directly next to the $\mathrm{B}$ component, so there may be some physical continuation between the cloud and the B component.

\section{ALMA results: spectral lines}

The observations covered several molecular emission features, which are listed in Table 1 and shown in a composite spectrum in Fig. 2. All observed lines are pure rotational transitions involving the ground vibrational levels. Their carriers are common oxides - including $\mathrm{SiO}, \mathrm{CO}, \mathrm{SO}$ (along with ${ }^{34} \mathrm{SO}$ ), and $\mathrm{SO}_{2}$ - and $\mathrm{H}_{2} \mathrm{~S}$. Millimeter-wave emission of these molecules has been accounted for in V838 Mon since the SMA observations of Kamiński et al. (2018). We additionally tentatively detected rare isotopologs of ${ }^{13} \mathrm{CO}$ and ${ }^{29} \mathrm{SiS}$. A newly detected species is $\mathrm{AlOH}$ which is observed through its $J=7-6$ transition (Apponi et al. 1993) and is seen in the spectral range that was not covered by the earlier SMA observations. This species is blended with a flank of the ${ }^{13} \mathrm{CO} 2-1$ line, if this latter is genuinely present.

Physical conditions in the molecular gas surrounding V838 Mon were studied by Kamiński et al. (2018). There, a local thermal equilibrium (LTE) excitation analysis was based on SMA observations of multiple lines observed in a much wider spectral range than covered here with ALMA. As our new ALMA observations provide the actual sizes of the emission regions, we revised the multi-line models by implementing updated beam-filling factors. Aside from changes in the absolute values of column densities, we obtained very similar results to our previous ones. The gas has source-averaged brightness temperatures of between around 70 and $200 \mathrm{~K}$ for different molecular tracers. Although ALMA spatially resolved the source, we are unable to investigate temperature gradients through line ratios because for each species ALMA covered only a single transition. However, our radiative transfer calculations show that the emission of CO $2-1$ is moderately optically thick $(\tau \approx 1)$ at line cores at positions with strong emission. The emission is optically thin in line wings and most other positions. Brightness temperatures (per synthesized beam) of CO 2-1 are of 30-170 K. Assuming a beam-filling factor of one everywhere and given the moderate optical depth of the emission, this range is a rough measure of excitation temperatures at the optically thick surface of the CO region. We find column densities per beam of $10^{20} \mathrm{~cm}^{-2}$ or lower. These values are slightly higher than in Kamiński et al. (2018), but they yield an overall mass of $\mathrm{H}_{2}$ of $0.1 M_{\odot}$ which is consistent with the earlier data.

The ALMA data do not warrant good measurements of the ratios of column densities for $\mathrm{CO}$ isotopologs, even though ${ }^{12} \mathrm{CO}$,
Table 1. Lines observed in V838 Mon.

\begin{tabular}{|c|c|c|c|c|}
\hline Line & $\begin{array}{c}v \\
(\mathrm{GHz})\end{array}$ & $\begin{array}{c}E_{\mathrm{u}} \\
(\mathrm{K})\end{array}$ & $\begin{array}{c}A_{\mathrm{ud}} \\
\left(\mathrm{s}^{-1}\right)\end{array}$ & $g_{\mathrm{u}}$ \\
\hline${ }^{34} \mathrm{SO} ? 5_{6}-4_{5}$ & 215.8399 & 34.38 & $1.26 \mathrm{e}-4$ & 13 \\
\hline $\mathrm{SO}_{2} 22_{2,20}-22_{1,21}$ & $216.6433^{(a)}$ & 248.44 & $9.27 \mathrm{e}-5$ & 45 \\
\hline $\mathrm{H}_{2} \mathrm{~S} 22_{2,0}-2_{1,1}$ & $216.7104^{(a)}$ & 83.98 & $4.87 \mathrm{e}-5$ & 5 \\
\hline $\mathrm{SiO} 5-4$ & 217.1049 & 31.26 & $5.20 \mathrm{e}-4$ & 11 \\
\hline $\mathrm{SO}_{2} 22_{7,15}-23_{6,18}$ & 219.276 & 352.76 & $2.13 e-5$ & 45 \\
\hline $\mathrm{SO} 5_{6}-4_{5}$ & 219.9494 & 34.98 & $1.34 \mathrm{e}-4$ & 13 \\
\hline $\mathrm{AlOH} 7-6$ & $220.3344^{(a)}$ & 42.30 & $6.18 \mathrm{e}-6$ & 12 \\
\hline${ }^{13} \mathrm{CO} ? 2-1$ & $220.3987^{(a)}$ & 15.87 & $6.08 \mathrm{e}-7$ & 10 \\
\hline $\mathrm{CO} 2-1$ & 230.538 & 16.60 & $6.91 \mathrm{e}-7$ & 5 \\
\hline${ }^{29} \mathrm{SiS} ? 13-12$ & 231.6267 & 77.82 & $2.10 \mathrm{e}-4$ & 27 \\
\hline
\end{tabular}

Notes. Frequencies $(v)$, upper energy levels $\left(E_{\mathrm{u}}\right)$, Einstein coefficients for spontaneous emission $\left(A_{\mathrm{ud}}\right)$, and statistical weights $\left(g_{\mathrm{u}}\right)$ are given. Question marks indicate uncertain identification. All transitions are withing the ground vibration level of the respective molecule. ${ }^{(a)}$ Blended.

${ }^{13} \mathrm{CO}$, and $\mathrm{C}^{18} \mathrm{O}$ were covered (cf. Fig. 2). Nevertheless, by simulating the spectra under LTE, we find a line ratio ${ }^{12} \mathrm{CO} /{ }^{13} \mathrm{CO}$ of larger than about 40. This ratio is hard to constrain because the weaker ${ }^{13} \mathrm{CO}$ line blends with the wider and much stronger $\mathrm{AlOH} 7-6$ line. The $\mathrm{C}^{18} \mathrm{O}$ is not detected, yielding only an upper limit on the $\mathrm{CO} / \mathrm{C}^{18} \mathrm{O}$ ratio of about 80 . These limits are consistent with earlier but equally uncertain constraints of ${ }^{12} \mathrm{CO} /{ }^{13} \mathrm{CO} \approx 10-100$ (Geballe et al. 2007).

Interferometric maps obtained with ALMA reveal detailed distributions of emission for the five strongest spectral features; three of which, namely CO (2-1), SiO (5-4), and SO $\left(5_{6}-4_{5}\right)$, are transitions that are relatively uncontaminated by emission of other species. We use the three lines to trace the spatiokinematical distribution of the gas. The upper energy levels $\left(E_{\mathrm{u}}\right)$ for the transitions are, respectively, 16.6, 31.3, and $35.0 \mathrm{~K}$. The maps of velocity-integrated emission (zeroth moment) are shown for all the species in Fig. 3. Channel maps for the $\mathrm{SiO}$ and $\mathrm{CO}$ emission are shown in Appendix C. The distribution of gas emission is different from that of the dust continuum emission. To illustrate variations of lines profiles within the different regions defined by continuum emission, in Fig. 4 we present representative spectra of $\mathrm{CO}, \mathrm{SiO}, \mathrm{SO}$, and $\mathrm{AlOH}$ at the continuum peak positions of components $\mathrm{M}$ and $\mathrm{B}$, and at the center of the extended cloud.

We assume that $\mathrm{CO}$ is the most representative probe of the entire molecular remnant, that is, it can be used as a proxy for the $\mathrm{H}_{2}$ distribution. The $\mathrm{CO}$ emission is the most extended and is fairly symmetric (Fig. 3). On maps reconstructed with natural weighting, the $5 \sigma$ contour extends up to a radius of $115 \pm 5$ mas or $679 \pm 30$ au from the V838 Mon position, except for directions near to the position of the companion where $\mathrm{CO}$ extends farther, up to a radius of 170 mas. The maximum $\mathrm{CO}$ emission is not at the position of V838 Mon but is about 20 mas southeast of that position. In maps obtained with uniform weighting, the emission appears clumpy, but none of the brightest CO clumps coincide with the positions of the stars (Fig. 5).

Emission of the SiO 5-4 line is almost as bright and extended as that of CO 2-1. On total intensity maps, however, the entire northwestern part of the ejecta beyond a radius of $\approx 80$ mas is 

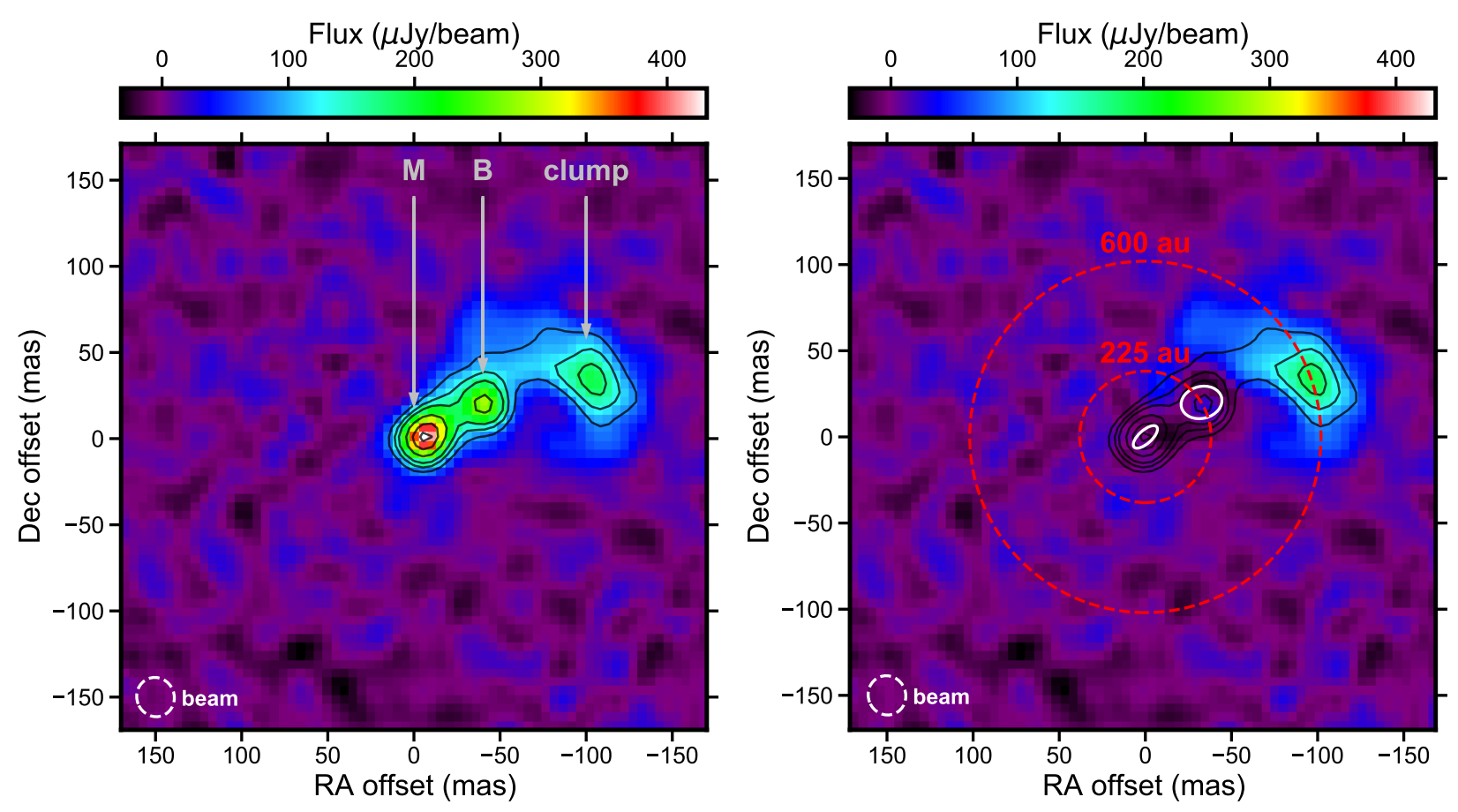

Fig. 1. Millimeter-wave continuum emission of V838 Mon. Left: ALMA map at $1.3 \mathrm{~mm}$ with all discussed sources labeled. Right: residual emission after subtracting Gaussian fits to components M and B. Ellipses drawn with a full line show the FWHM of the fitted Gaussian components. White ellipses drawn with a dashed line show the FWHM of the restoring beam. Two red large circles in the right panel are centered on the position of component $\mathrm{M}$ and are drawn for radii of 225 and $600 \mathrm{au}$; they illustrate the characteristic physical scales of the remnant (see Sect. 3). Contours in both images show the original continuum emission at $0.2,0.3,0.4,0.6,0.8$, and 0.95 times the peak emission. The maps have the same color scale. The angular offsets are with respect to the best-fit central position of the $\mathrm{M}$ component.
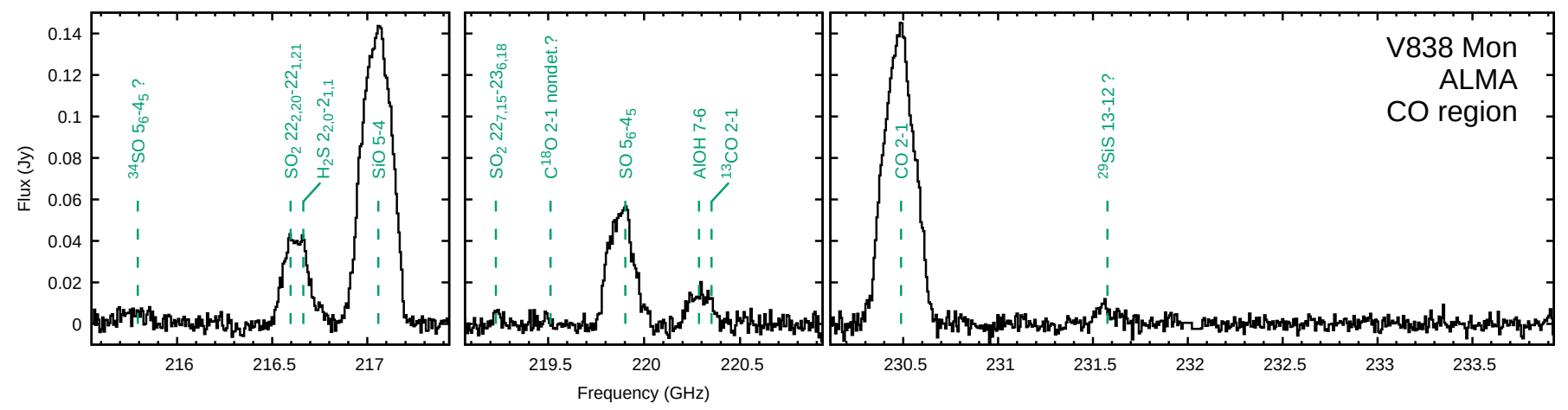

Fig. 2. ALMA spectrum of V838 Mon representing the entire emission region of CO. Main identified features are labeled and marked with dashed lines at frequencies corresponding to the systemic velocity. Uncertain identifications are indicated with a question mark.

missing when compared to $\mathrm{CO}$. This is especially apparent in the channel maps (see Appendix $\mathrm{C}$ ). The region with missing $\mathrm{SiO}$ partially overlaps with the extended dust clump. The brightest $\mathrm{SiO}$ emission is, as in the case of $\mathrm{CO}$, arising from areas that are away from V838 Mon, but bright $\mathrm{CO}$ and $\mathrm{SiO}$ clumps do not overlap.

Despite similar values of $E_{\mathrm{u}}$ for the observed $\mathrm{SO}$ and $\mathrm{SiO}$ lines, the distribution of emission is significantly different for the two species. The SO emission is concentrated northeast from the position of V838 Mon and only weak emission is seen west of component M. A similar emission distribution is seen in the corresponding line of ${ }^{34} \mathrm{SO}$, but at a much lower $\mathrm{S} / \mathrm{N}$. Part of the differences between $\mathrm{SO}$ and $\mathrm{SiO}$ distributions may be caused by a higher optical depth of the $\mathrm{SiO}$ line. However, we believe that most of the differences arise because of intrinsic changes in the chemical composition within the molecular remnant.
The blend of AlOH 7-6 and ${ }^{13} \mathrm{CO} 2-1$ is dominated by emission of the $\mathrm{AlOH}$ line. Although its map has a modest $\mathrm{S} / \mathrm{N}$, it shows a clumpy emission region whose overall size is smaller than the ${ }^{12} \mathrm{CO}$ region. Also, unlike $\mathrm{CO}$, the $\mathrm{AlOH}$ emission peaks northwest of V838 Mon, between dust B component and the dust clump. The emission region corresponding to the blend of $\mathrm{SO}_{2}$ and $\mathrm{H}_{2} \mathrm{~S}$ has very similar characteristics to the emission region of $\mathrm{AlOH}$. The excitation of the $\mathrm{AlOH}$ transition (with $E_{\mathrm{u}}$ of $42.3 \mathrm{~K}$ ) requires much lower temperatures than the lines of $\mathrm{SO}_{2}$ and $\mathrm{H}_{2} \mathrm{~S}$ (248.4 and $84 \mathrm{~K}$, respectively), strongly suggesting that the observed similarities in the location for the three species reflect gas chemical composition, and not excitation.

In the lower right panel of Fig. 3 we also present a map produced from panchromatic visibilities from which the continuum emission was subtracted as a polynomial fit. This map represents combined emission of all spectral lines present in 



Fig. 3. Total intensity maps of the molecular emission in V838 Mon. A white contour is drawn at the $3 \sigma$ noise level of each map. Gray contours show continuum emission at $0.2,0.3,0.4,0.6,0.8$, and 0.95 times the peak level. All maps were reconstructed with natural weighting. Bottom left and middle panels: maps for blended transitions. The energy of the upper level of the mapped transitions is indicated on each map. Bottom right panel: emission in all lines covered by ALMA. The red dashed circle of a radius of 750 au serves as a reference for the physical scale.

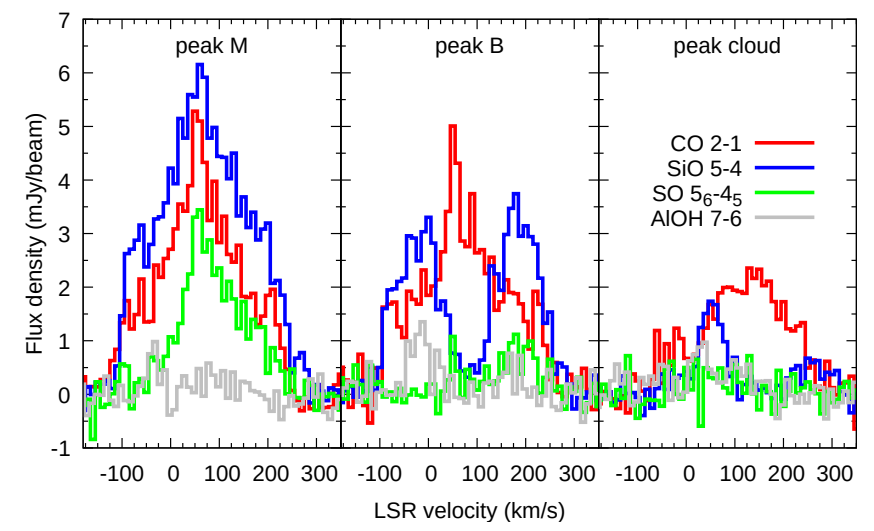

Fig. 4. Spectra of the main species at the three continuum peaks of the remnant. Each represents a single beam.

the band, although is clearly dominated by the $\mathrm{CO}$ and $\mathrm{SiO}$ emission. Owing to a higher sensitivity, the map shows more clearly that the molecular envelope has the largest extent in directions close to the lines connecting the three continuum sources. The $3 \sigma$ contour reaches out to a radius of 184 mas or
$1086 \mathrm{au}$. It is also apparent that the outer outline of the molecular region is not smooth, indicating a substructure possibly related to a form of hydrodynamical instability, possibly of the KelvinHelmholtz type. After performing simulations in the CASA task simobserve, we are confident the irregular outlines of the emission regions are not an instrumental effect but are real features. The CASA test was performed by producing visibility data for the same baselines as those used in real observations and for an ideal elliptical source. After imaging, the simulated observations yield a much smoother source than observed in molecular emission of V838 Mon.

The velocity structure of the molecular cloud is complex. We illustrate it in Fig. 6 with first-moment maps. Overall, the southeastern part of the envelope is more blueshifted than the northwestern part. The maps certainly do not show the signature expected for an expanding and nonrotating spherical shell. With respect to the systemic LSR velocity of V838 Mon $\left(54 \mathrm{~km} \mathrm{~s}^{-1}\right.$; Kamiński et al. 2018), significantly higher speeds are observed in the deciding (redshifted) part than in the approaching one. Highly redshifted gas is seen mostly in the northwestern part of the envelope and close to its outer edges. Emission of SO probes only a small part of the envelope, but also shows an excess of redshifted gas in the western part. 

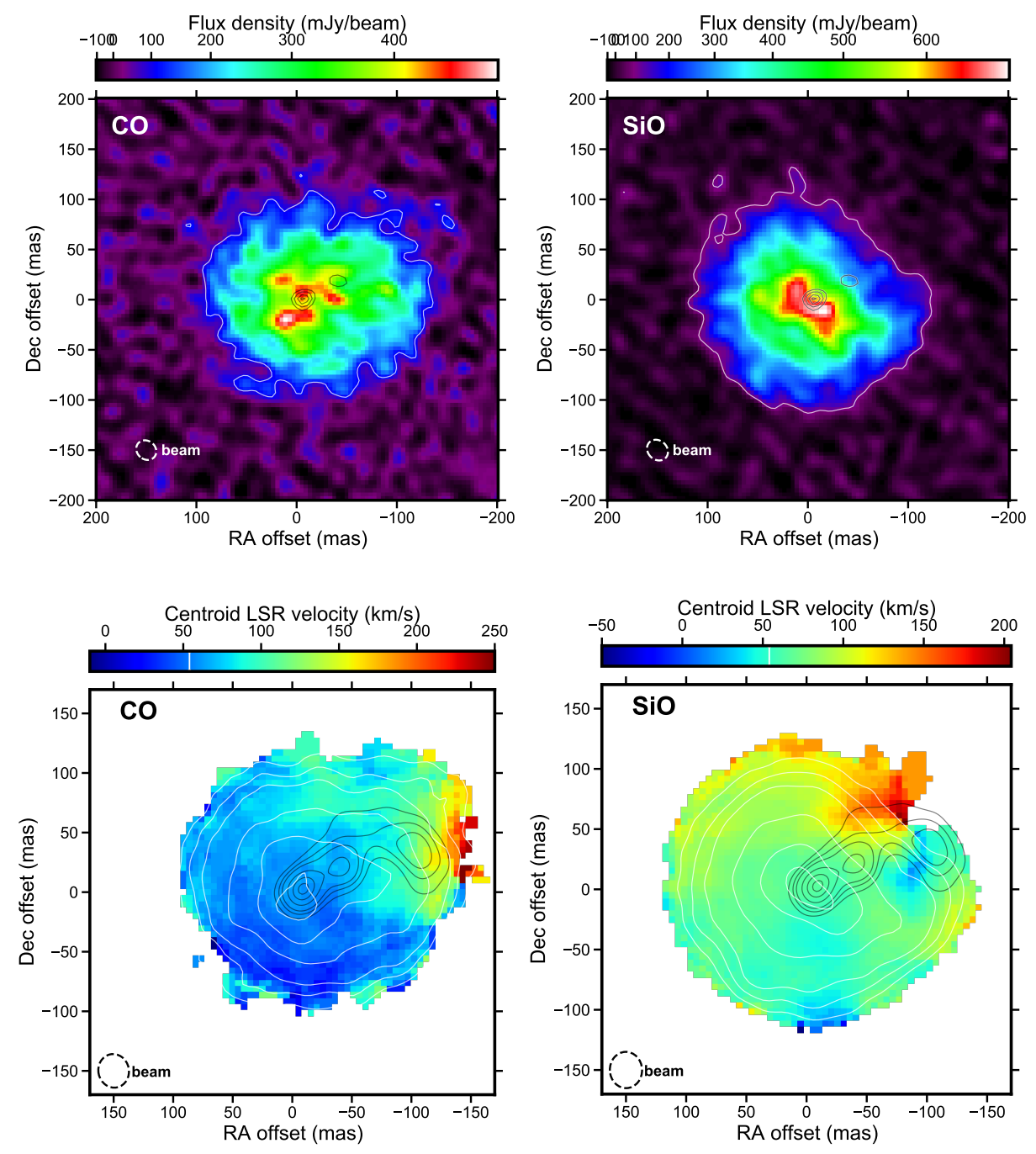

Fig. 5. As in Fig. 3 but for $\mathrm{CO}$ and $\mathrm{SiO}$ at uniform weighting of visibilities. Continuum contours, also at uniform weighting, start at 0.3 times peak level.

Fig. 6. First-moment maps of unblended molecular lines. White contours show the total intensity of any given line. Gray contours show continuum emission. Contours are drawn at 0.2, 0.3, 0.4, 0.6, 0.8, and 0.95 times the peak flux. The white line at the colorbar marks the stellar (systemic) velocity of V838 Mon. The maps were produced with natural weighting of visibilities. The moment maps were calculated after masking all pixels with total intensity below the $5 \sigma$ map noise level.

The envelope kinematics can also be characterized through second-moment maps, which show dispersion in radial velocity. The maps shown in Fig. 7 combine the dispersion caused by the Doppler effect arising in the projected envelope and the dispersion intrinsic to the gas velocity field. For $\mathrm{CO}$ and $\mathrm{SiO}$, circular symmetry is broken again in the western or northwestern part of the second-moment maps. Assuming that most of the observed dispersion arises from the Doppler effect and that the envelope has a roughly symmetric structure, the excess dispersion of about $30 \mathrm{~km} \mathrm{~s}^{-1}$ in the western and northwestern envelope can be interpreted as a measure of excessive gas motions, possibly caused by stronger turbulence. The region with the largest dispersion seen in $\mathrm{SiO}$ coincides with the location of the strongest emission of the $\mathrm{SO}_{2}+\mathrm{H}_{2} \mathrm{~S}$ blend (see the right panel of Fig. 7).

\section{Optical spectroscopy near the ALMA epoch}

Here we briefly present optical spectra of V838 Mon from 2012 and 2020, which bracket the epoch of our ALMA observations. They are complementary to the ALMA data because they present information about the supergiant star and its circumstellar medium on the line of sight to the bright star. While a full description and presentation of the spectra will be given in a dedicated paper, we provide basic information about the optical spectra in Appendix A. The spectral evolution of the object at optical wavelengths prior to 2012 is discussed in depth in Loebman et al. (2015), Kamiński et al. (2009), Tylenda et al. (2011b) and references therein.

Our flux-calibrated broadband X-shooter spectrum from 2012 was used for spectral typing. The spectrum was first compared to catalog spectra of red giants and red supergiants with known spectral types and in the spectral regions least affected by the thick circumstellar envelope of V838 Mon. A remarkably good match was found with SV* HV 1963, a Li-rich AGB star in the Small Magellanic Cloud. Its spectrum was found in the X-shooter Spectral Library (Gonneau et al. 2020). It is classified as M4 Ib-II (after González-Fernández et al. 2015), but detailed models of the star yield $T_{\text {eff }}=3300-3350 \mathrm{~K}$ and $\log g=-$ 0.027 to 0.0 and $[\mathrm{Fe} / \mathrm{H}]=-0.5$ (Plez et al. 1993; Kiselman \& Plez 1995). We next compared the spectrum of V838 Mon to synthetic MARCS spectra (Gustafsson et al. 2008) and found a good correspondence between $3300 \mathrm{~K}$ stellar models and molecular 

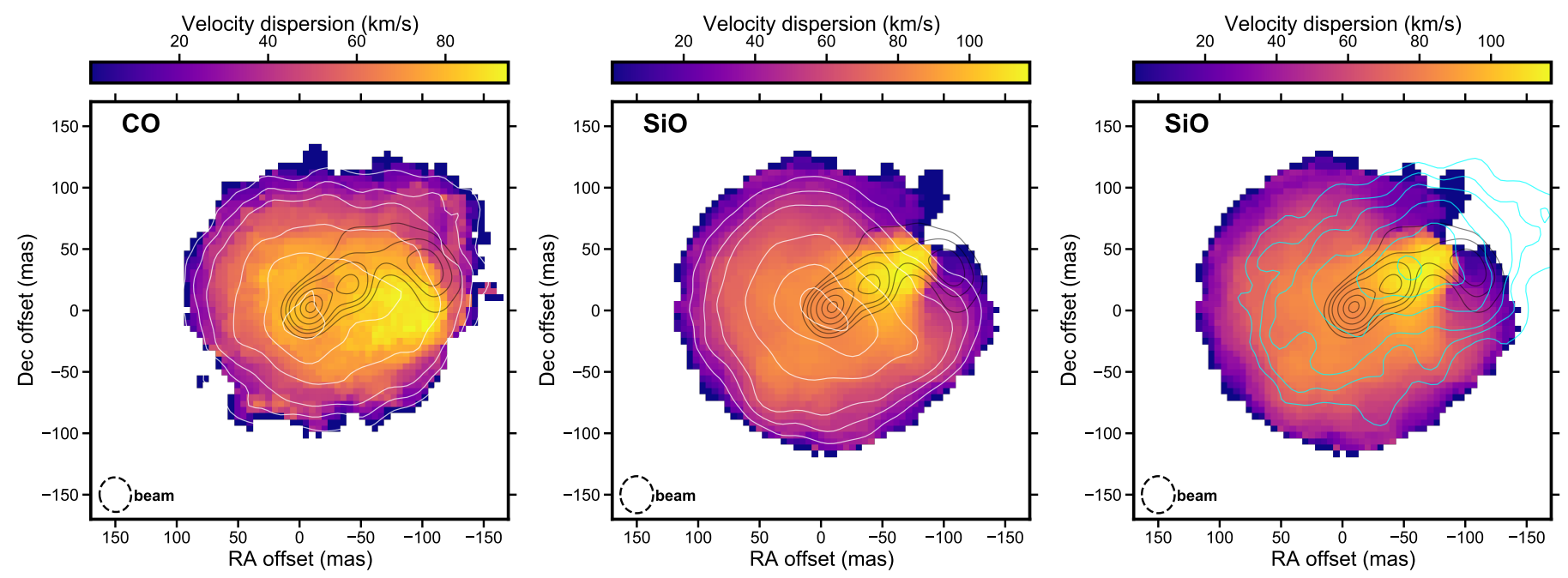

Fig. 7. Second-moment maps for molecular lines of $\mathrm{CO} 2-1$ and $\mathrm{SiO} 5-4$. White contours show the total intensity of any given line. Gray contours show continuum emission. Cyan contours in the right panel show total intensity of the $\mathrm{SO}_{2}$ and $\mathrm{H}_{2} \mathrm{~S}$ blend. Contours are drawn at 0.2, 0.3, 0.4, 0.6, 0.8 , and 0.95 times the peak flux. The maps were produced with natural weighting of visibilities.

bands of V838 Mon originating from the excited vibrational or electronic states. The same comparison to synthetic spectra was performed for the 2020 spectrum from SALT. It yielded a slightly higher photospheric temperature of $3500 \mathrm{~K}$, and could suggest a slow rise in the photospheric temperature of V838 Mon over the last decade or so. These temperatures are significantly higher than the 2000-2200 K claimed by Loebman et al. (2015) and based on optical and infrared (IR) spectra obtained a few years earlier. It is possible that the earlier study did not exclude spectral regions dominated by molecular absorption of cool nonphotospheric gas and that therefore their estimate does not correspond to the photosphere of the star. The temperatures of 3300 and $3500 \mathrm{~K}$ are more consistent with those of Kamiński et al. (2009) $(3200 \mathrm{~K})$ and Tylenda et al. (2009) $(3270 \mathrm{~K})$ in earlier epochs (2005 and 2009, respectively).

Our spectra show strong signatures of the cool circumstellar matter on the line of sight, mainly in lines of neutral alkali metals and in molecular bands. Sample atomic profiles for four epochs are shown in Fig. 8. The strongest atomic lines are resonance transitions of abundant species, such as the optical doublets of $\mathrm{KI}$ and $\mathrm{Na}$ I, or the $\lambda 5110$ line of Fe I. These are the broadest absorption features spreading from the Local Standard of Rest (LSR) systemic velocity of the star of $54 \mathrm{~km} \mathrm{~s}^{-1}$ (cf. Kamiński et al. 2018) down to LSR velocities of $\approx-150 \mathrm{~km} \mathrm{~s}^{-1}$ (or from 71 to $-132 \mathrm{~km} \mathrm{~s}^{-1}$ in the heliocentric frame). The deep absorption lines have not changed significantly over recent years. In weaker atomic lines, the same velocity range is occupied by several narrower features which become weaker with time, as illustrated in Fig. 8 in the profiles of $\mathrm{Rb}$ I $\lambda 7800$ and $\mathrm{Ca}$ I $\lambda$ 6572. The absorption has completely disappeared in some species in the most recent spectrum, for instance in the $\mathrm{Rb}$ I line. The continuing fainting of the weaker absorption lines, known from earlier spectroscopic monitoring of V838 Mon, shows primarily the expanding merger ejecta that becomes more diluted and cooler with time. Absorption components at lower radial velocities, close to the systemic one, represent an ongoing wind originating in the supergiant-like stellar remnant. In the 2020 SALT spectrum lines from highly excited states, such as lines of Ti I from a $0.8 \mathrm{eV}$ state, display a relatively narrow absorption component at a heliocentric velocity of $+85 \mathrm{~km} \mathrm{~s}^{-1}$ indicative of an infall. Its location and origin is unclear, but it is very likely it coexists with the wind component in the immediate vicinity of the photosphere.

Among all atomic features, the profile of $\mathrm{H} \alpha$ is unique (Fig. 8, top). It shows a weak inverse P-Cyg type profile with the absorption component centered nearly at the systemic velocity. The $\mathrm{H} \alpha$ emission peaks near an LSR velocity of about $5 \mathrm{~km} \mathrm{~s}^{-1}$. The origin of the $\mathrm{H} \alpha$ feature has been unclear, but it was speculated it may arise in the medium photoionized by the B star (Loebman et al. 2015; Tylenda et al. 2011b). Our recent spectra show it is a persistent feature on time scales longer than a decade and is relatively constant both in position and in intensity.

Overall, referring to gas ejected in the merger event, the optical spectra from before and after the epoch of ALMA observations show the remnant has been in a similar state since around 2005 , that is, since the disappearance of the B star in the visual and since pure emission lines vanished.

\section{Three-dimensional reconstruction of the remnant}

\subsection{Dust}

We attempted a reconstruction of the dust distribution in the remnant of V838 Mon using RADMC-3D ${ }^{1}$ (Dullemond et al. 2012). For a given configuration of dust density distribution and location of radiation sources, the dust temperature is calculated using the radiative equilibrium assumption. Next, a ray-tracing method is used to simulate images and integrated fluxes of the system at different wavelengths. We compared the simulations to a spectral energy distribution (SED) of the entire remnant of V838 Mon (all components) ranging from the $U$ optical band to the mm band of ALMA. The data sources for the observed SED are briefly described in Appendix B. Simultaneously, we tried to reproduce the spatial features seen in the ALMA maps.

Even in the simplest considered configuration, the modeled system has numerous free parameters. They cannot be uniquely constrained by our observations and therefore the model we

\footnotetext{
1 https://www.ita.uni-heidelberg.de/ dullemond/ software/radmc-3d/index. php
} 


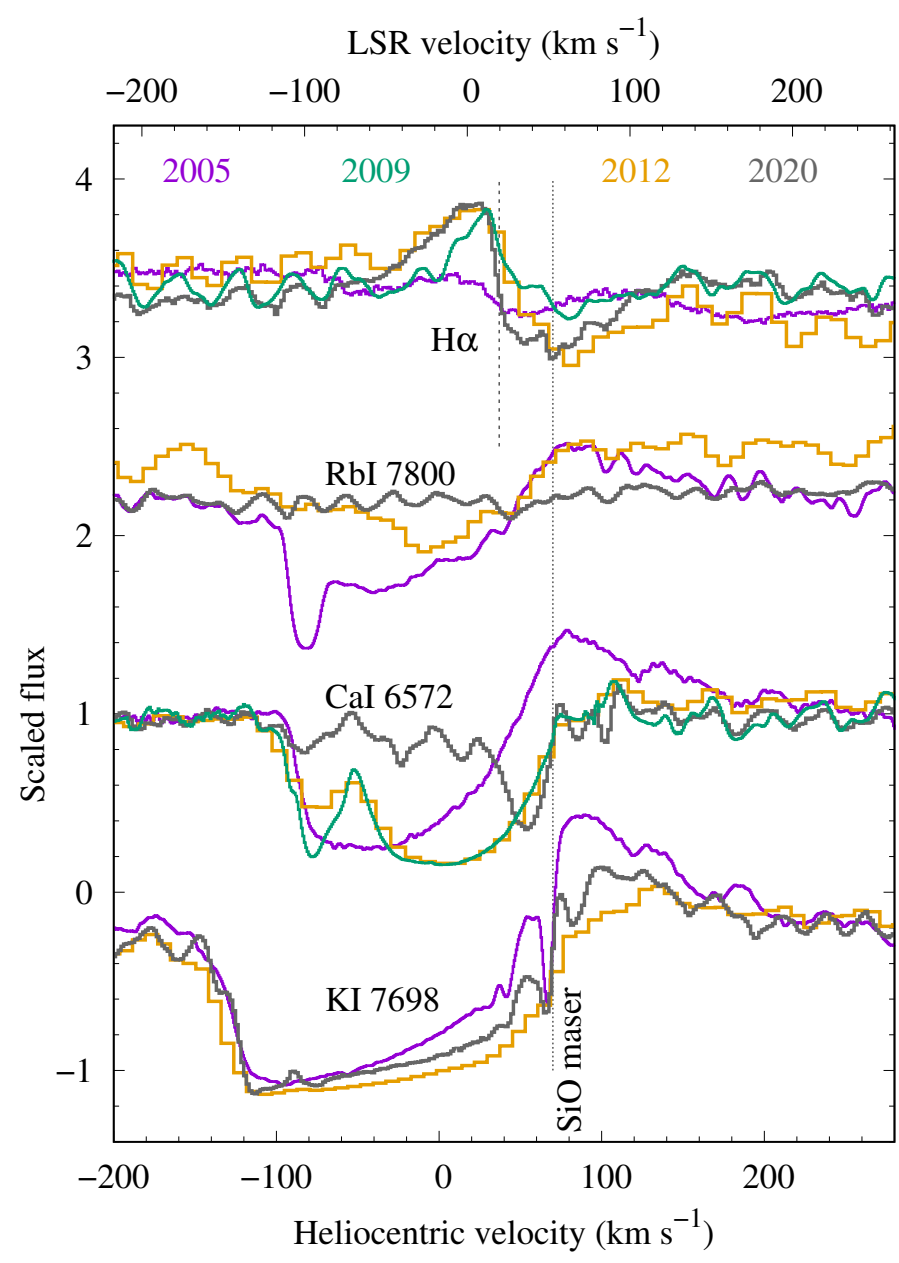

Fig. 8. Temporal changes in selected atomic line profiles of V838 Mon. Keck/HIRES spectra from 2005 (Kamiński et al. 2009) are shown in purple, VLT/UVES spectra from 2009 in green (Tylenda et al. 2011b), VLT/X-shooter spectra from 2012 in orange, and SALT/HRS spectra from 2020 in gray. The spectra were arbitrarily scaled to show changes in the four labeled atomic lines. The vertical lines mark the velocity of the $\mathrm{SiO}$ maser (or the supposed stellar systemic velocity; $54 \mathrm{~km} \mathrm{~s}^{-1} \mathrm{LSR}$ or $71 \mathrm{~km} \mathrm{~s}^{-1}$ in the heliocentric frame) and the position of the turnover in the inverse P-Cyg profile of $\mathrm{H} \alpha\left(37 \mathrm{~km} \mathrm{~s}^{-1}\right.$ heliocentric).

attempted to match to the observations was rather exploratory. After running thousands of simulations, we arrived at a model described below and shown in Fig. 9. A comparison between observed and simulated images and SEDs is shown in Figs. 10 and 11 .

The only heat sources in the system are the two stars. The companion was represented by a black body of a temperature of $18 \mathrm{kK}$, a radius of $3.1 \times 10^{11} \mathrm{~cm}\left(4.5 R_{\odot}\right)$, and a luminosity of $1.9 \times 10^{3} L_{\odot}$. These values correspond to a spectral type of B3 V. We located the blue star in the sky plane at 230 au away from V838 Mon that is at the same distance along the line of sight as V838 Mon. V838 Mon was represented by a black body of a fixed temperature of $3300 \mathrm{~K}$, consistent with spectral typing performed on the most recent optical and IR spectra of the star (Sect. 5). The radius and thus luminosity of V838 Mon were adjusted to match the SED. The optimal values we arrived at are $3.25 \times 10^{13} \mathrm{~cm}\left(464 R_{\odot}\right)$ for the radius and $2.3 \times 10^{4} L_{\odot}$ for the luminosity.

The biggest uncertainty in the SED reconstruction comes from dust opacities as a function of wavelength and location in

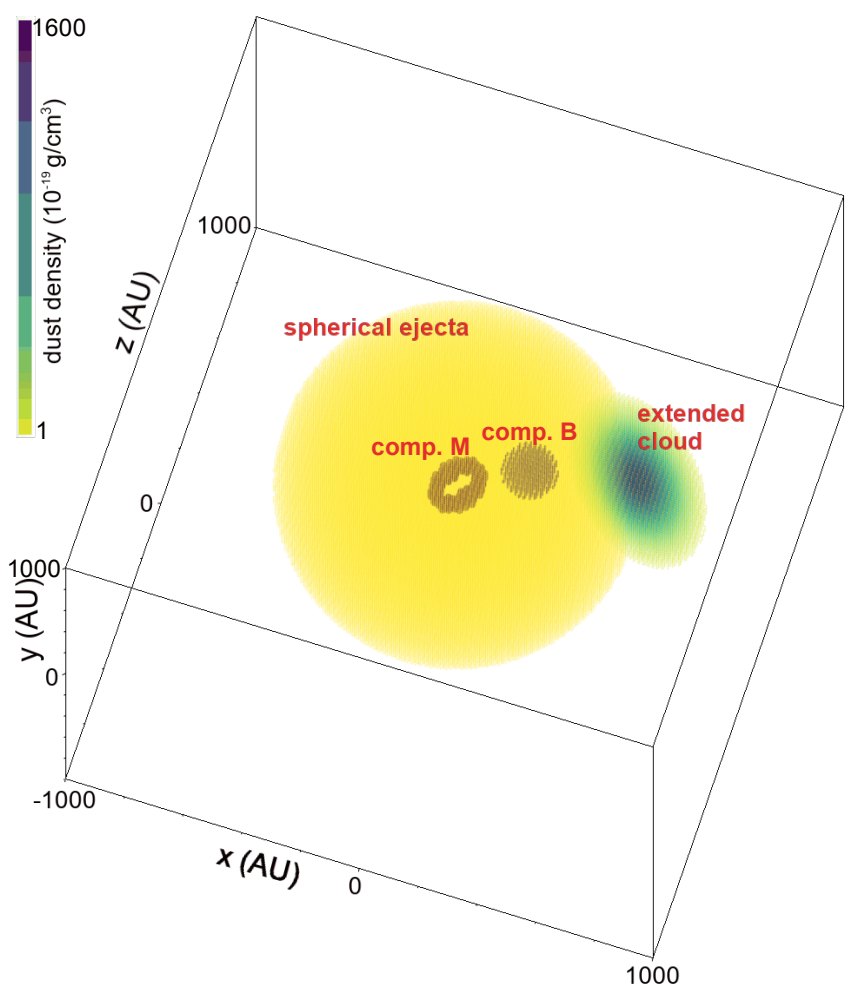

Fig. 9. Three-dimensional model of dust distribution in V838 Mon in 2019. An animation presenting the model is available online. Model grid points are represented by semi-transparent spheres whose size and color correspond to local dust density. The color scale is linear from $1.0 \times$ $10^{-19} \mathrm{~g} \mathrm{~cm}^{-3}$ (yellow) to $1.6 \times 10^{-16} \mathrm{~g} \mathrm{~cm}^{-3}$ (dark purple). The sky plane is for $x=0$ and $y=0$ and the $z$ axis is along the line of sight. The volume shown is a cube of $2000 \mathrm{au}$ in height, length, and breadth.
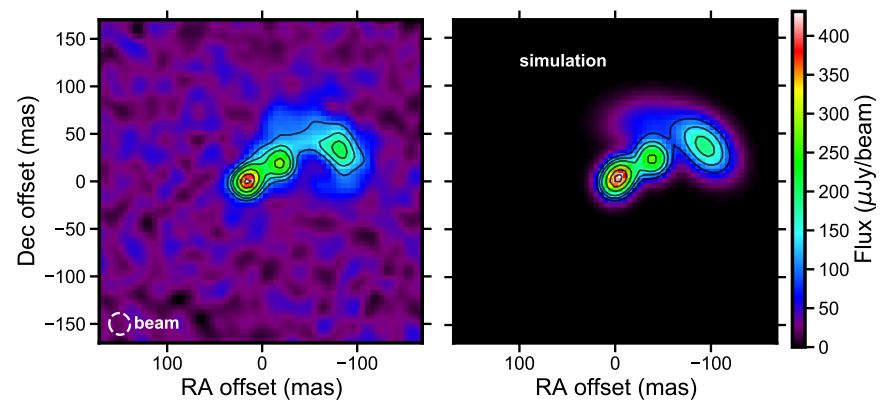

Fig. 10. Comparison of observed (left) and simulated (right) maps of continuum emission in ALMA band 6. Both images are displayed with the same color scale, contour levels, and angular resolution.

the remnant. As the gas seen in absorption and emission spectra is oxygen-rich (i.e., it is indicative of a higher abundance of oxygen than of carbon), a form of silicate dust is expected to dominate in the opacity curve. We arbitrarily implemented an opacity curve of Mg-rich silicates (amorphous pyroxene with 70\% Mg-rich and 30\% Fe-rich) compiled from data in Jaeger et al. (1994) and Dorschner et al. (1995) and for a single grain size of $0.1 \mu \mathrm{m}$. Grain density was set to $3 \mathrm{~g} \mathrm{~cm}^{-3}$. This opacity curve matches the IR part of the SED near $10 \mu \mathrm{m}$ slightly better than most other silicate opacities frequently used in the modeling of circumstellar media. Material in the real remnant probably comprises a range of dust species with different grain 


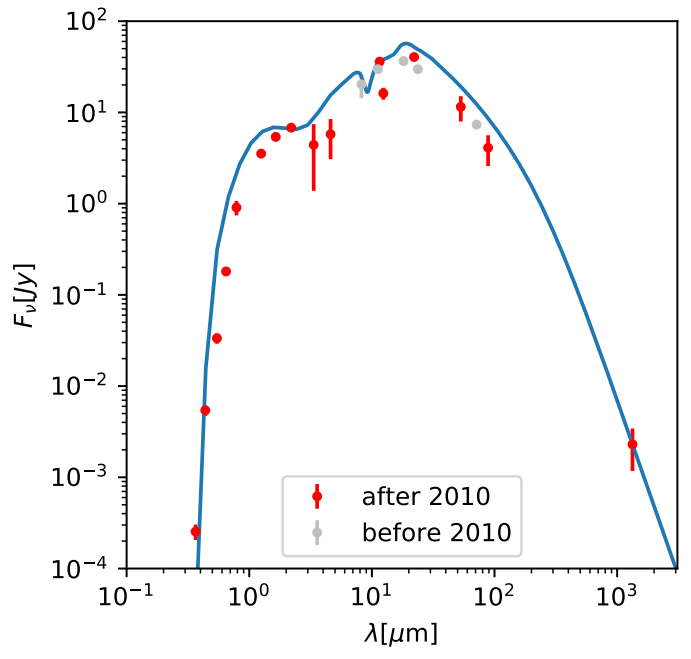

Fig. 11. Comparison of the observed (points) and simulated (line) SED. Uncertainties in dust opacities are much larger than the apparent discrepancies between the observations and the simulation.

sizes and compositions, but those cannot currently be uniquely constrained.

The simulated $\mathrm{M}$ component is a disk-like or torus-like structure immediately surrounding the stellar remnant of V838 Mon and has a dust mass of $9.4 \times 10^{-4} M_{\odot}$. It was implemented as a structure filled with dust for a range of azimuthal angles, $65^{\circ}<\theta<115^{\circ}$, and whose plane is inclined to the line of sight by $26^{\circ}$ so that the disk edge does not obscure the central star for our line of sight. The structure has a maximal radius of $95 \mathrm{au}$. It is hollow in the center with a hole radius of $60 \mathrm{au}$. At the outer hole radius, the dust temperature is $1350 \mathrm{~K}$. The hole can be interpreted as a clearing related to the sublimation of dust. When inclined, such an elongated structure nicely explains the shape of the beam-deconvolved component M (Sect. 2; Fig. 3). It is also consistent with VLTI observations of the region at MIR wavelengths (Chesneau et al. 2014). The dust density was assumed to increase with distance from V838 Mon as $r^{0.75}$. We also attempted to reproduce the $\mathrm{M}$ component by a spherical dusty cloud, such as one expected from a spherically expanding supergiant wind. However, such a configuration results in a higher extinction towards the central star than observed. We favor models with a flattened structure, such as a disk or a torus.

External to the disk, we place a spherical shell at radii of between 95 and $600 \mathrm{au}$, which we refer to as a mergeburst shell. Although extended, this structure was implemented to have a low dust density on the order of $10^{-19} \mathrm{~g} \mathrm{~cm}^{-3}$ which only slightly increases with distance (as $r^{0.1}$ ). The dust mass of the structure is rather low, $8.0 \times 10^{-5} M_{\odot}$. We associate the shell with the merger ejecta directly seen in the molecular emission traced by ALMA and seen in absorption lines of neutral metals and of simple molecules at optical wavelengths throughout the entire post-outburst evolution of the object (cf. Sect. 5). In the model, the spherical mergeburst shell is the only source of extinction on the line of sight of the cool star, with $A_{V} \approx 1 \mathrm{mag}$.

We surround the blue star with a shell of dust of constant density (for simplicity) of $1.5 \times 10^{-16} \mathrm{~g} \mathrm{~cm}^{-3}$ and extending from 10 to $95 \mathrm{au}$ from the hot star. Again, the inner clearing is caused by dust sublimation. The outer radius is constrained by the beam-deconvolved size of component $\mathrm{B}$. We require this extra shell in our model to produce the mm-wave source, but it
Table 2. Modeled dust Components of V838 Mon.

\begin{tabular}{|c|c|c|c|}
\hline Component & Form & $\begin{array}{l}\text { Mass } \\
\left(M_{\odot}\right)\end{array}$ & $\begin{array}{c}\text { Extent } \\
\text { (au) }\end{array}$ \\
\hline Component $\mathrm{M}$ & disk-like & $9.4 \mathrm{e}-4$ & $60-95$ \\
\hline Component B & sph. shell & $9.4 \mathrm{e}-4$ & $10-95$ \\
\hline Spherical ejecta & sph. shell & $8.0 \mathrm{e}-5$ & $95-600$ \\
\hline Eextended cloud & 3D Gauss. & $6.1 \mathrm{e}-3$ & $\begin{array}{l}(150 \times 95 \times 119)^{(a)} \\
(220 \times 350 \times 277)^{(a)}\end{array}$ \\
\hline
\end{tabular}

Notes. ${ }^{(a)}$ FWHM

is not clear whether the dusty structure is limited to a spherical region directly surrounding the $\mathrm{B}$ star or is part of a more extended component whose parts, not illuminated by the stars, are not visible in the map. In principle, we could increase the local density of the mergeburst shell so that it produces the observed mm signature around star B, but then the extinction toward the red star becomes much higher, becoming inconsistent with visual observations. Alternatively, a flattened structure surrounding V838 Mon and overlapping the orbit of the B star may be responsible for component $\mathrm{B}$. The dust mass of the small shell surrounding only the B star is $9.4 \times 10^{-4} M_{\odot}$, but if component $\mathrm{B}$ is associated with extended but dark dust, the actual mass may be much higher.

As the last component of the simulation, we added two Gaussian-shaped clouds to reproduce the extended $\mathrm{mm}$ continuum emission north and northwest of the B component. Gaussian shapes are chosen to ease the implementation, and we only aimed at reproducing the main features of the extended component. (Reproducing the ALMA images precisely for this extended component would be trivial by adding extra components.) We locate the two representative clouds in the sky plane, that is, in the same plane. The relative location of the components is important, as the shell surrounding component B casts a shadow on the two Gaussian extended clouds, partially blocking light from the bright cool star. The clouds are only excited by photons of the two stars - no inner heat source, such as shocks, were implemented, although they may be adequate for this spatial component (see Sect. 7). The cumulative mass of the dispersed dust in these two clouds is $6.1 \times 10^{-3} M_{\odot}$. This mass is thus significantly higher than that of all the other components of the system. The high mass is required to produce the mm-wave fluxes in the absence of an internal heat source.

The masses and extents of the model components are summarized in Table 2 .

\subsection{Molecular gas}

We also attempted to reconstruct the distribution of molecular gas with RADMC-3D. Such models are however even less constrained than those for dust because gas models require extra input for the temperature and velocity structure (and thus even more free parameters). Although we were able to construct models that very satisfactorily reproduce maps and spectral profiles of CO observed by ALMA, the solutions we find are by no means unique or particularly revealing. Observations of at least one more transition of $\mathrm{CO}$ or $\mathrm{SiO}$ at a similar angular resolution would lift many of the model degeneracies. For $\mathrm{CO}$, a single, almost spherical shell with temperature, density, and radial velocity gradients given as power laws was sufficient to explain most of the observational characteristics. We also found that $\mathrm{CO}$ 


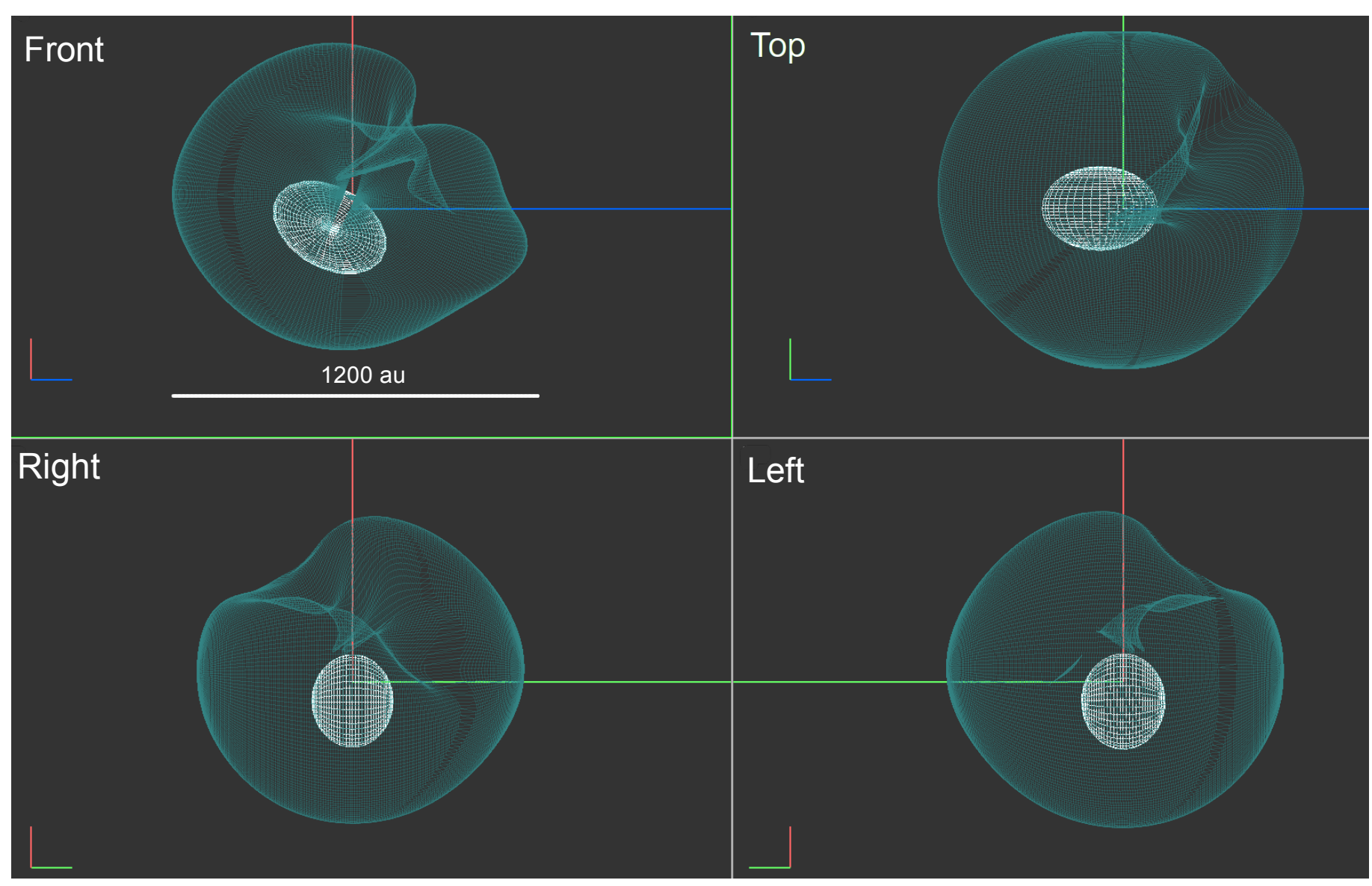

Fig. 12. Model of the spatial extent of SiO emission around V838 Mon. The mesh structures show two regions with different velocity fields and density (or intensity) power laws which reproduce channel maps and position-velocity diagrams of the SiO line. The outer shell contains a characteristic void which the model attempts to reproduce. The "front" view (top left) corresponds to that of the terrestrial observer. The model origin corresponds to the centroid position of component M. An animation is provided online.

line profiles are better reproduced with the systemic velocity of $+70 \mathrm{~km} \mathrm{~s}^{-1}$ rather than $54 \mathrm{~km} \mathrm{~s}^{-1}$ defined by the $\mathrm{SiO}$ maser near V838 Mon (Deguchi et al. 2005).

To investigate the possible structure of the outflow in molecular tracers other than CO, we used the Shape software ${ }^{2}$ (Steffen et al. 2011), which gives more flexibility in constructing complex spatial models than RADMC-3D. We focused on reproducing the observations in the optically thin limit with very simplified radiative transfer. Here we only present the results for the $\mathrm{SiO}$ transition. This required two shell-like structures, each with a different density profile (or rather intensity) and slightly different velocity fields. The latter were implemented as simple relations with radial velocity increasing linearly with distance $(v=k r)$. The velocity gradient is steeper for the outer structure $(k=1.9)$ than for the inner one $(k=1.7)$. The inner shell was best reproduced as an elliptical structure whose center is slightly shifted with respect to the position of component $\mathrm{M}$. The larger structure is more spherically symmetric, but required the introduction of a cone-shaped void in the northwestern part. The rather complex shape of the larger structure is presented in Fig. 12. The void seems to extend from the position of the B component and grows in size with distance from the geometrical center. Our simple model satisfactorily reproduces structures in channel maps, shown in Appendix $\mathrm{C}$, and position-velocity diagrams but no attempt to reproduce absolute intensities was undertaken in this case. The model strongly suggests that the void is directly related

2 https://wsteffen75. wixsite.com/website to the passage of matter near component $\mathrm{B}$, but does not shed light on whether it is caused by depletion of $\mathrm{SiO}$ or by a change in excitation conditions.

\section{Discussion}

The ALMA view on the 2019 remnant clarifies the architecture of the system $17 \mathrm{yr}$ after the merger and for the first time directly shows the location of the B3 V companion. The different system components identified in the observations and through the modeling nevertheless pose several additional problems. These are discussed below, first for the gaseous molecular component.

\subsection{Molecular gas in the remnant of V838 Mon}

Historical view. So far, most of the information about the circumstellar environment of the V838 Mon system has come from single-line optical and MIR spectroscopy. During the outburst, the maximal outflow velocities traced in atomic features were about $500 \mathrm{~km} \mathrm{~s}^{-1}$ (Kipper \& Škoda 2007; Wisniewski et al. 2003) and the outflow velocities were considerably variable over the three months of the outburst (Munari et al. 2002b; Kipper et al. 2004; Kipper \& Škoda 2007; Wisniewski et al. 2003; Rushton et al. 2005; Crause et al. 2003). Broad absorption lines from cool atomic and molecular gas have been observed since the end of the eruption, first as part of P-Cygni profiles (Kipper et al. 2004; Kipper \& Škoda 2007; Kamiński et al. 2009; 
Tylenda et al. 2009; Geballe et al. 2007; Lynch et al. 2004; Wisniewski et al. 2003) and later on, after around 2006, mainly as pure absorption features (Loebman et al. 2015; Kamiński et al. 2018; Geballe et al. 2007). The absorption troughs indicated maximal outflow velocities of $250 \mathrm{~km} \mathrm{~s}^{-1}$ (Kamiński et al. 2009; Tylenda et al. 2009) but most of the material was moving at lower speeds. In particular, Tylenda et al. (2009) found three slower atomic components, with radial velocities of about 50, 110, and $150 \mathrm{~km} \mathrm{~s}^{-1}$, and Geballe et al. (2007) found CO components at $15,85,150 \mathrm{~km} \mathrm{~s}^{-1}$. Since the gas ejection velocities were evidently changing during the eruption, the remnant was assumed to be stratified and spherically symmetric models with homogeneous and isothermal shells were adopted for the circumstellar molecular gas (Lynch et al. 2004, 2007; Loebman et al. 2015). Presenting maps of the molecular emission for the first time, we can conclude that the ejecta is not as stratified as it is clumpy (Fig. 5). Thus, absorption spectroscopy alone, limited to one line of sight, does not provide a full picture of the entire remnant.

Wind. Spectral signatures at optical and $\mathrm{mm}$ wavelengths reveal an ongoing wind that is emanating from the central red supergiant. Based on the most recent optical high-resolution spectra from the SALT telescope (Sect. 5), the terminal velocity of the wind is $\$ 50 \mathrm{~km} \mathrm{~s}^{-1}$ (cf. Tylenda et al. 2009; Ortiz-León et al. 2020; Kamiński et al. 2018). Assuming that the wind took the present form in mid 2002 and freely expanded over the $17 \mathrm{yr}$ preceding the ALMA epoch, it should have reached a radius of $179 \mathrm{au}$ or 30 mas, which constitutes one-third of the size of the remnant traced by $\mathrm{CO}$ emission in the ALMA epoch (see right panel of Fig. 3). If this is correct, the wind has not yet reached the orbit of the companion. The $\mathrm{SiO}$ maser, which appears to gradually drop in intensity (Deguchi et al. 2005; Ortiz-León et al. 2020), is excited in the innermost regions of this clumpy wind. The emission of different species traced by ALMA, especially emission of SO (Fig. 3), reveals excitation variations or chemical inhomogeneities within the wind, not unlike those known from genuine red supergiants (Kamiński et al. 2013; Adande et al. 2013; Montargès et al. 2019). As the stellar remnant is expected to be highly magnetic (Soker \& Tylenda 2007; Schneider et al. 2020), perhaps some of these asymmetries can be linked to magnetism.

Merger ejecta. ALMA provides the clearest view so far of the molecular merger ejecta. The molecular gas seen in $\mathrm{CO}$ ro-vibrational absorption bands had the highest velocities of $\approx 190 \mathrm{~km} \mathrm{~s}^{-1}$ (Geballe et al. 2007), much lower than those observed in atomic gas. The warm molecular component at this speed should have reached a radius of $700 \mathrm{au}$ or 119 mas in the ALMA epoch. This size is in excellent agreement with the observed tangential extent of the rotational mm-wave $\mathrm{CO}$ emission, that is, to a nominal radius of 115 mas (Fig. 3). This suggests that the tangential motions of the bulk of the molecular gas are of the same magnitude as those along the line of sight. Thus, the ejecta is roughly spherical. The full width of the CO $J=2-1$ line profile representing the entire molecular cloud mapped by ALMA indicates maximal radial velocities of $230 \pm 20 \mathrm{~km} \mathrm{~s}^{-1}$, which are higher than those seen in the IR. This high-velocity gas is seen as weak emission in the line wings and has very low surface brightness, meaning that it could have been missed in the IR observations.

Unseen atomic gas. Assuming spherical symmetry and ignoring the wind component, the merger ejecta should have taken the form of an expanding shell whose outer parts contain the fastest ejecta and the inner ones have the lowest outflow velocities associated with the eruption. The fastest ejecta (at $500 \mathrm{~km} \mathrm{~s}^{-1}$ ) should have reached a distance of 1840 au or 311 mas in mid 2019, over twice farther away than any molecular or dust tracer seen by ALMA. Our observations therefore show us only the inner ( $\sim$ half) part of the gaseous remnant. Observations with an instrument capable of resolving atomic or dust-scattered emission at scales of 600 mas would be necessary to probe the high-velocity ejecta. This should be possible, for instance, with the SPHERE instrument on the VLT. Unfortunately, emission lines are currently very weak in V838 Mon (Sect. 5; see also Loebman et al. 2015).

Interaction with the $B$ star. Gas kinematics revealed by ALMA show the northwestern region in the vicinity of the B component to be the most turbulent and the most redshifted portion of the remnant, suggestive of gas interactions with the B star. The possible nature of the interaction is discussed in Sect. 7.3. The different molecular tracers observed by ALMA also reveal chemical composition variations within the inner remnant (SO vs. CO) and in the outer merger ejecta. These variations are most pronounced near (or, from the perspective of V838 Mon, "behind") the B companion. Although there is a remote chance that the observed differences in emission distribution are related to different excitation conditions, we find it more likely that this turbulent region hosts shocks that strongly influence gas molecular composition ${ }^{3}$. Some species appear to have enhanced abundances in the interaction region, including $\mathrm{SO}_{2} / \mathrm{H}_{2} \mathrm{~S}$ and $\mathrm{AlOH}$. Others, like $\mathrm{SiO}$, are depleted (Sect. 6.2). In astrochemical studies of the interstellar medium (ISM), S-bearing species are often assumed to trace shocks (Pineau des Forets et al. 1993; Feng et al. 2020), corroborating our interpretation. The interaction of the merger ejecta with the B-type star and its environment poses an interesting case for circumstellar shock-induced chemistry worthy of further investigation. If shock chemistry were influencing the chemistry in this interaction region, then the lack of $\mathrm{SiO}$ might appear surprising at first glance. As in the seminal work of Schilke et al. (1997), an enhanced $\mathrm{SiO}$ abundance is recognized as a tracer of shocks in the ISM. These authors ascribe an enhancement of the $\mathrm{SiO}$ abundance, which is generally low in the ISM, to the sputtering of silicate dust grains, a process in which atomic silicon is set free and oxidized.

Location of the $B$ star. Let us assume that the asymmetry in the first-moment maps of molecular emission in Fig. 6 is caused by interaction of the outflow with the companion. In this case, the redshifted gas can be interpreted as part of the material that was slingshot away from us by the gravity of the B star. Redshifted material then indicates that the companion is not exactly in the plane of the sky, but is more distant than V838 Mon. Detailed modeling would be necessary to test this scenario and constrain the relative distance of the two stars along the line of sight. An earlier study of the relative location of the stars by Tylenda et al. (2009) suggested an opposite configuration. Based on the profiles of the [Fe II] emission lines, it was calculated that the photoionized material was slightly blueshifted with respect to

\footnotetext{
3 We combined SMA spectra of $\mathrm{SO}_{2}$ from Kamiński et al. (2018) with the ALMA line, and used nondetections of $\mathrm{SO}_{2}$ transitions requiring high excitation to construct a simple LTE model of the emission. Although blending with $\mathrm{H}_{2} \mathrm{~S}$ prevents very precise deductions, we concluded that the $\mathrm{SO}_{2}$ regions cannot be much warmer than those characterizing other species at the same projected distances from V838 Mon. Then, the maps of molecular emission are best explained by abundance variations.
} 
V838 Mon in the initial phases of the engulfment. More specifically, an angle of $10-12^{\circ}$ from the sky plane was found, placing the B star slightly closer to us than V838 Mon. Overall, for now it is safest to assume that both stars are in the sky plane (as we did in our 3D modeling).

\subsection{Dusty remnant}

The continuum emission components surrounding the stars can be easily explained as thermal dust emission of grains heated by the radiation of the two stars (Sect. 6.1). Although it would be tempting to assume that the dust has the same spatial distribution as the molecular gas, this is evidently not the case in V838 Mon (Fig. 3).

The merger remnant is surrounded by a wind that may have a dust component. The emission of this component must be optically thin and cannot be easily identified in the observations. Our modeling shows that the elongated component M surrounding the stellar remnant is most likely a torus-like structure seen at a moderate inclination (Sect. 6.1). Although this interpretation is not firm, as our data lack the angular resolution needed for a definitive conclusion, such a structure is expected to exist around merged systems. Also, disks are observed in other rednova remnants (Kamiński \& Tylenda 2013; Tylenda et al. 2011a). Such a disk or a torus-like structure is expected to carry most of the angular momentum of the former binary and consist of the common envelope material. Future interferometric observations in the IR may measure that angular momentum and thereby constrain the common envelope physics.

The nature of component $\mathrm{B}$ is easy to understand at first sight. ALMA maps identify the position of the B star and leave no doubt that the star forms a physical system with V838 Mon. Assuming that the B star is at a circular orbit of a size of $230 \mathrm{au}$ in a system of a mass of $8.0+8.4 M_{\odot}$, its orbital period is $1000 \mathrm{yr}$ and the orbital velocity is $3.8 \mathrm{~km} \mathrm{~s}^{-1}$. Such a wide binary of two intermediate-mass stars is expected to be eccentric (Tokovinin 2020), but there is currently no way to constrain this orbital parameter. As mentioned before, from the timing of the interaction of the merger ejecta with the B star (Tylenda et al. 2009), it seems that the plane of the orbit must be very close to the sky plane, but the asymmetry in profiles of molecular lines suggests that the B star is slightly more distant than V838 Mon. It would be interesting to know whether or not the real orbit is coplanar with the disk found in component $\mathbf{M}$ and, if not, as seems to be the case, what role the Kozai-Lidov cycles (Kozai 1962; Lidov 1962) played in the merger of the inner binary (cf. Naoz \& Fabrycky 2014).

It is natural to assume that the dust giving rise to the mmwave emission of component B comes from the dusty merger ejecta encircling the B star. However, our radiative transfer modeling suggests that, at least for a spherically symmetric merger ejecta, component $\mathrm{B}$ requires an enhanced dust density to produce the observed mm signature (Sect. 6.1). Alternatively, it is possible that there is a ring of dust overlapping with the orbit of the B star, not crossing the line of sight, and whose major part is dark for us. Another possibility is that some micro-physical processes increase dust condensation only near the hot star, for instance, due to a collision of the ejecta with the wind of the B star (cf. Usov 1991). This would partially explain the depletion of $\mathrm{SiO}$ (Sect. 6.2), which in this scenario would be easily incorporated into solid material. Yet another interpretation is that some material was captured from the flow and remains bound to the star. Nevertheless, this scenario can be excluded based on the considerations outlined in Appendix D. Future observations of the eventual re-appearance of the B star in the optical may shed more light on the origin of component B.

The third continuum source, that is the extended northeastern cloud, is most difficult to explain, especially because there is no distinct corresponding counterpart in the $\mathrm{CO}$ emission. (A simple partial overlap with $\mathrm{CO}$ in the sky plane may, in general, be coincidental.) This third source either requires a strongly enhanced dust density for a range of azimuthal angles in the outer remnant or involves an inner dispersed heat source, such as shocks and their radiation. In either case, some special conditions must have prevailed in the northwestern part of the remnant. The location of the extended component is close to the line connecting V838 Mon with component B, leading us to believe that it is the interaction of the ejecta with the B star that created the extended cloud.

\subsection{Interaction of the merger ejecta with the $B$ star}

\subsubsection{Wind accretion (deflection)}

The ejecta passing the B companion interacts gravitationally with the star, resulting in a geometrical configuration typically described as wind accretion of the Bondi-Hoyle-Lyttleton (BHL) type (Edgar 2004). Assuming the average ejecta speeds of the molecular and dusty outflow of $150 \mathrm{~km} \mathrm{~s}^{-1}$ (Sect. 7.1), the accretion radius (or Bondi-Hoyle radius) is $0.6 \mathrm{au}$ or 27 times the radius of the $\mathrm{B}$ star. In the absence of other effects, material with impact parameters smaller than this radius will be accreted in the wake of the flow through an unstable accretion column (Ohsugi 2018) (but see below); particles with slightly larger impact parameters will be deflected (gravitationally focused) and may collide with each other in the wake of the flow, producing a turbulent region upstream. The region with a high velocity dispersion and with altered composition of molecular gas seen in our ALMA maps near the companion is most naturally explained by this effect. Some particles are accelerated passing by the B star and this is perhaps why we observe the larger extent of the molecular remnant along the line connecting the two stars (see bottom right panel in Fig. 3).

Although we invoke the BHL scenario here, we do not claim that there is significant ongoing accretion onto the B star. From $\mathrm{CO}$ observations we estimate that the $\mathrm{H}_{2}$ density near the $\mathrm{B}$ star is about $10^{6} \mathrm{~cm}^{-3}$ which gives accretion rates of about $10^{-10} M_{\odot} \mathrm{yr}^{-1}$. This is only an approximate value, calculated under multiple simplifying Bondi-Hoyle assumptions, but it implies a very low accretion luminosity, of $\approx 0.006 L_{\odot}$, which is insignificant for the system energetics.

Looking at the continuum maps (Fig. 1), it is tempting to conclude that the curving tail of continuum emission is a continuous feature emanating from V838 Mon and being sculpted by the gravity of the companion. The orbital motion of the companion of $\approx 4 \mathrm{~km} \mathrm{~s}^{-1}$ is much slower than the ejecta, and therefore it does not cause any significant or systematic acceleration of the ejecta in directions other than the original flow motion. However, if our estimate of the orbital period of $1000 \mathrm{yr}$ is roughly correct and the orbit is circular and very close to the sky plane, the B star has moved by $6^{\circ}$ since the eruption (with respect to the position of V838 Mon). This is schematically illustrated in the sketch in Fig. 13. Assuming arbitrarily that the B star moves counterclockwise, the bright part of the extended dusty cloud was closer to the line connecting the stars $17 \mathrm{yr}$ ago than it was in the ALMA epoch. If the interaction with the B star is responsible for the outer dusty cloud, it had to involve material that was lost from the inner system years prior to the eruption. (The 


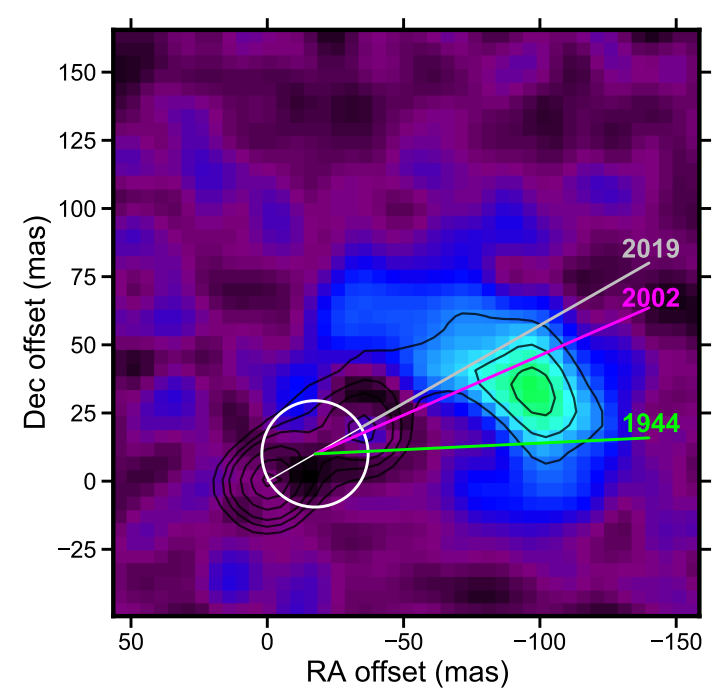

Fig. 13. Same as the residual map in Fig. 1 but showing a hypothetical circular orbit (white circle) of the wide binary. Assuming a clockwise motion for the B star, the dusty clump was on the line connecting the two stars in the recent past.

outer dust cloud, V838 Mon, and the B star were all aligned near 1990, over a decade before the merger; see Fig. 13.) Although mass loss is expected in systems evolving into a merger years before the final coalescence (e.g., Tylenda et al. 2011a; MacLeod et al. 2018; Pejcha et al. 2016a,b), assigning the outer dusty cloud to such a phenomenon is currently highly speculative. Also, it is unclear why the cloud is seen only in dust emission and not in gas tracers.

\subsubsection{Ionization, radiation pressure, and wind of the B star}

In 2005, the ejecta - then approaching the B star - was readily photo-ionized by radiation of the hot star (Tylenda et al. 2009). That process very likely continued beyond 2005 , but eventually the ejecta became too optically thick for the ionizing photons to penetrate deep into the outflow. The H II region must currently have a small volume and in the optical is completely obscured by dust of the B component.

The radiation of the $\mathrm{B}$ star provides significant pressure acting on the dust of the ejecta. Assuming a full collisional momentum coupling between dust and molecular gas, we estimate in Appendix D that the acceleration caused by radiation of an unattenuated B3 V star may even be ten times stronger than its gravity. As also discussed in Appendix D, however, sublimation of grains heated by stellar photons limits the expansive effect of radiation pressure, especially for small impact parameters. Once grains are destroyed, practically no momentum transfer from photons occurs (except for a weak effect in atomic lines). Overall, we expect that radiation pressure adds to the net scattering effect of material passing the B star and can be in part responsible for the relatively wide opening angle of the wake that is seen in the northwestern part of the remnant in the continuum maps. In the case of an inefficient collisional coupling between dust and gas in the ejecta, the scattering effect of radiation pressure should be stronger for dust.

The effect of radiation pressure acting on dust should produce a cone-like void behind the B star, very similar to that seen in $\mathrm{SiO}$ distribution. We modified the 3D model of the dust distribution presented in Sect. 6.1 by introducing a conical void whose apex is anchored at the B star, and which has an opening angle of $\sim 90^{\circ}$ and its central axis pointing to V838 Mon. The modification of the model has three main consequences. First, the density of dust in the remaining part of the envelope surrounding the B star needs to be increased by about $30 \%$ to match the flux of the ALMA observations. Second, although the B component still appears roughly circular, introducing the void shifts its centroid position $\sim 4$ mas closer to V838 Mon. To reproduce the observations, the separation thus needs to be increased to $250 \mathrm{au}$. Third, the clearing around the B star exposes the external dust clouds to the unattenuated radiation of the B star and consequently $20 \%$ less dust is needed to explain the observed flux levels of the extended cloud.

In addition to the radiation pressure effect, one could expect a repelling effect from a wind of the B star. Early B-type stars on the main sequence have tenuous winds with mass-loss rates on the order of $10^{-11}-10^{-10} M_{\odot} \mathrm{yr}^{-1}$ and terminal velocities of 800 $1330 \mathrm{~km} \mathrm{~s}^{-1}$ (Krtička 2014; Cohen et al. 1997). As we show in Appendix E, such a wind will stop and shock the merger ejecta, but its dynamical effect on the shape of the flow wake is negligible. Collisions of wind and ejecta particles are expected to be quite energetic in V838 Mon, but there are currently no observations that would directly identify the associated shock signatures in the environment of the red nova (Antonini et al. 2010). Nevertheless, such shocks may provide an extra local radiation source, heating dust in the zone where the two outflows collide.

Given that nearly $17 \mathrm{yr}$ have passed since the eruption (in the ALMA epoch) and $15 \mathrm{yr}$ since the engulfment of the B star by the dusty ejecta, we would expect the companion to reappear for optical observations - most of the ejecta has passed the companion and become geometrically thinner on our line of sight owing to the expansion. The reappearance has not yet happened (Goranskij et al. 2020) either because the dusty ejecta has a large radial extent, which is unlikely, or part of the dusty material that obscured the B star has become gravitationally bound. The latter would be surprising in face of the physical mechanisms discussed above which should clear the dust from the immediate surroundings of the B star. Time-dependent models would be necessary to investigate this problem further.

\section{Summary}

We report ALMA observations of the merger site of V838 Mon $17 \mathrm{yr}$ after its outburst and at an unprecedented resolution corresponding to spatial scales of $150 \mathrm{au}$. Continuum dust emission and emission in several molecular transitions were mapped and analyzed with radiative transfer calculations. The highresolution maps directly show the complex interplay between material ejected during the red nova eruption and the remaining binary system. The inner remnant is dominated by a clumpy molecular wind similar to circumstellar envelopes of genuine red supergiants. Images of mm-wave continuum, supported by $3 \mathrm{D}$ radiative-transfer modeling, provide new evidence of a dusty elongated structure of about 95 au in the intermediate neighborhood of the stellar remnant of V838 Mon. The structure may be a dusty disk that stores the angular momentum of the binary that merged in 2002 and thereby is most interesting for studies of stellar mergers and common envelope events. Future observations of the disk require IR interferometric techniques.

The mm-wave continuum maps show, for the first time, the location of the B star, which has remained embedded in the dusty ejecta since 2005 and is not visible at shorter wavelengths. The orbit of the B star around V838 Mon has a size of $\gtrsim 230$ au and, 
assuming a circular case, has a period of about $1000 \mathrm{yr}$. These parameters make the V838 Mon system most similar to Antares $\mathrm{AB}$, where a wind of a red supergiant interacts with a $\mathrm{B} 2.5 \mathrm{~V}$ star at a similarly wide orbit. Instead of the slow $\left(20 \mathrm{~km} \mathrm{~s}^{-1}\right)$ wind of Antares A, in the case of the V838 Mon system the hot star interacts with the much thicker and faster $\left(150 \mathrm{~km} \mathrm{~s}^{-1}\right)$ ejecta produced in the 2002 eruption of the red nova. This interaction will likely continue in the future, allowing us to better constrain the orbit of the B star and investigate whether it could have triggered the merger of the inner binary through the Kozai-Lidov mechanism (Naoz \& Fabrycky 2014).

The ALMA data reveal a complex interaction of the gravity and radiation of the hot star with the overflowing ejecta of V838 Mon. The gravitational effect of the B star accelerates the ejecta and creates a turbulent wake, which we identify in ALMA data as a region with an increased velocity dispersion and a changing chemical composition. Associated shocks create conditions where some species are enhanced $\left(\mathrm{SO}_{2} / \mathrm{H}_{2} \mathrm{~S}\right)$ while others become depleted $(\mathrm{SiO})$, presenting a unique laboratory of shockinduced circumstellar chemistry. The radiation pressure deflects part of the ejecta into a wide-angle cone and imposes differences in the distribution of gas and dust in the outer remnant.

Comparing our ALMA data to the results of spectroscopic monitoring of the object at shorter wavelengths, we find that external to the molecular remnant described here is a fast atomic outflow not seen at mm wavelengths but able to be mapped with modern instruments at optical or IR wavelengths. Such observations would be necessary to assess the entire mass of the circumstellar material. Without accounting for the unknown contribution of the atomic phase, the estimate of the mass of the remnant is currently $M_{\mathrm{H} 2}=0.1 M_{\odot}$. Our independent but uncertain estimate of the mass of the dust alone is $8.3 \times 10^{-3} M_{\odot}$. The total mass and future characterization of the angular momentum stored in the inner disk will provide invaluable parameters for constraining the merger scenario of V838 Mon and merger physics in general.

Acknowledgements. We thank Morgan MacLeod for discussions on possible interaction mechanisms in a wide binary of V838 Mon. T.K., A.K., and K L. acknowledge funding from grant no 2018/30/E/ST9/00398 from the Polish National Science Center. R.T. and A.F. acknowledge a support from grant 2017/27/B/ST9/01128 financed by the Polish National Science Center. The research work at the Physical Research Laboratory is funded by the Department of Space, Government of India. M.S. acknowledges a support by the Nationa Science Center, Poland, under grant 2016/21/B/ST09/01626. This paper makes use of the following ALMA data: ADS/JAO.ALMA\#2018.1.00336.S. ALMA is a partnership of ESO (representing its member states), NSF (USA) and NINS (Japan), together with NRC (Canada), MOST and ASIAA (Taiwan), and KASI (Republic of Korea), in cooperation with the Republic of Chile. The Joint ALMA Observatory is operated by ESO, AUI/NRAO and NAOJ. The National Radio Astronomy Observatory is a facility of the National Science Foundation operated under cooperative agreement by Associated Universities, Inc. Some observations reported in this paper were obtained with the Southern African Large Telescope (SALT). Polish participation in SALT is funded by grant No. MNiSW DIR/WK/2016/07. Based on observations collected at the European Organization for Astronomical Research in the Southern Hemisphere under ESO program 088.D-0112(A). This research is based in part on data collected at Subaru Telescope, which is operated by the National Astronomical Observatory of Japan. We are honored and grateful for the opportunity of observing the Universe from Maunakea, which has the cultural, historical and natural significance in Hawaii. Based in part on observations made with the NASA/DLR Stratospheric Observatory for Infrared Astronomy (SOFIA). SOFIA is jointly operated by the Universities Space Research Association, Inc. (USRA), under NASA contract NNA17BF53C, and the Deutsches SOFIA Institut (DSI) under DLR contract 50 OK 0901 to the University of Stuttgart.

\section{References}

Adande, G. R., Edwards, J. L., \& Ziurys, L. M. 2013, ApJ, 778, 22 Afşar, M., \& Bond, H. E. 2007, AJ, 133, 387

Antonini, F., Montez, R. J., Kastner, J. H., et al. 2010, ApJ, 717, 795

Apponi, A. J., Barclay, W. L., J., \& Ziurys, L. M. 1993, ApJ, 414, L129 Bond, H. E., Henden, A., Levay, Z. G., et al. 2003, Nature, 422, 405 Chesneau, O., Millour, F., De Marco, O., et al. 2014, A\&A, 569, L3 Cohen, D. H., Cassinelli, J. P., \& MacFarlane, J. J. 1997, ApJ, 487, 867 Cowley, A. P. 1969, PASP, 81, 297

Crause, L. A., Lawson, W. A., Kilkenny, D., et al. 2003, MNRAS, 341, 785 Crawford, S. M., Still, M., Schellart, P., et al. 2010, SPIE Conf. Ser., 7737, 773725 Deguchi, S., Matsunaga, N., \& Fukushi, H. 2005, PASJ, 57, L25

Dorschner, J., Begemann, B., Henning, T., Jaeger, C., \& Mutschke, H. 1995, A\&A, 300, 503

Dullemond, C. P., Juhasz, A., Pohl, A., et al. 2012, Astrophysics Source Code Library [record ascl: 1202.015]

Edgar, R. 2004, New Astron. Rev., 48, 843

Evans, A., Geballe, T. R., Rushton, M. T., et al. 2003, MNRAS, 343, 1054

Feng, S., Codella, C., Ceccarelli, C., et al. 2020, ApJ, 896, 37

Geballe, T. R., Rushton, M. T., Eyres, S. P. S., et al. 2007, A\&A, 467, 269

Gonneau, A., Lyubenova, M., Lançon, A., et al. 2020, A\&A, 634, A133

González-Fernández, C., Dorda, R., Negueruela, I., \& Marco, A. 2015, A\&A, 578, A3

Goranskij, V. 2006, ATel, 964, 1

Goranskij, V. P., Barsukova, E. A., Burenkov, A. N., et al. 2020, Astrophys. Bull., 75,325

Gustafsson, B., Edvardsson, B., Eriksson, K., et al. 2008, A\&A, 486, 951

Harper, D. A., Runyan, M. C., Dowell, C. D., et al. 2018, J. Astron. Instrum., 7, 1840008

Jaeger, C., Mutschke, H., Begemann, B., Dorschner, J., \& Henning, T. 1994, A\&A, 292, 641

Kamiński, T. 2008, A\&A, 482, 803

Kamiński, T., \& Tylenda, R. 2013, A\&A, 558, A82

Kamiński, T., Schmidt, M., Tylenda, R., Konacki, M., \& Gromadzki, M. 2009, ApJS, 182, 33

Kamiński, T., Tylenda, R., \& Deguchi, S. 2011, A\&A, 529, A48

Kamiński, T., Gottlieb, C. A., Young, K. H., Menten, K. M., \& Patel, N. A. 2013, ApJS, 209, 38

Kamiński, T., Steffen, W., Tylenda, R., et al. 2018, A\&A, 617, A129

Kataza, H., Okamoto, Y., Takubo, S., et al. 2000, SPIE Conf. Ser., 4008, 1144

Kausch, W., Noll, S., Smette, A., et al. 2015, A\&A, 576, A78

Kimeswenger, S., Lederle, C., Schmeja, S., \& Armsdorfer, B. 2002, MNRAS, 336, L43

Kipper, T., \& Škoda, P. 2007, ASP Conf. Ser., 363, 231

Kipper, T., Klochkova, V. G., Annuk, K., et al. 2004, A\&A, 416, 1107

Kiselman, D., \& Plez, B. 1995, Mem. Soc. Astron. It., 66, 429

Kozai, Y. 1962, AJ, 67, 591

Krtička, J. 2014, A\&A, 564, A70

Lenzuni, P., Gail, H.-P., \& Henning, T. 1995, ApJ, 447, 848

Lidov, M. L. 1962, Planet. Space Sci., 9, 719

Loebman, S. R., Wisniewski, J. P., Schmidt, S. J., et al. 2015, AJ, 149, 17

Lynch, D. K., Rudy, R. J., Russell, R. W., et al. 2004, ApJ, 607, 460

Lynch, D. K., Rudy, R. J., Russell, R. W., et al. 2007, ASP Conf. Ser., 363, 39

MacLeod, M., Ostriker, E. C., \& Stone, J. M. 2018, ApJ, 868, 136

McMullin, J. P., Waters, B., Schiebel, D., Young, W., \& Golap, K. 2007, ASP Conf. Ser., 376, 127

Montargès, M., Homan, W., Keller, D., et al. 2019, MNRAS, 485, 2417

Munari, U., Desidera, S., \& Henden, A. 2002a, IAU Circ., 8005, 2

Munari, U., Henden, A., Kiyota, S., et al. 2002b, A\&A, 389, L51

Munari, U., Corradi, R. L. M., Henden, A., et al. 2007, A\&A, 474, 585

Naoz, S., \& Fabrycky, D. C. 2014, ApJ, 793, 137

Neugent, K. F., Levesque, E. M., Massey, P., \& Morrell, N. I. 2019, ApJ, 875, 124

Ohsugi, Y. 2018, Astron. Comput., 25, 44

Okamoto, Y. K., Kataza, H., Yamashita, T., et al. 2003, SPIE Conf. Ser., 4841, 169

Ortiz-León, G. N., Menten, K. M., Kamiński, T., et al. 2020, A\&A, 638, A17

Pavlenko, Y. V., van Loon, J. T., Evans, A., et al. 2006, A\&A, 460, 245

Pejcha, O., Metzger, B. D., \& Tomida, K. 2016a, MNRAS, 461, 2527

Pejcha, O., Metzger, B. D., \& Tomida, K. 2016b, MNRAS, 455, 4351

Pineau des Forets, G., Roueff, E., Schilke, P., \& Flower, D. R. 1993, MNRAS, 262, 915

Plez, B., Smith, V. V., \& Lambert, D. L. 1993, ApJ, 418, 812

Rushton, M. T., Geballe, T. R., Filippenko, A. V., et al. 2005, MNRAS, 360, 1281 
T. Kamiński et al.: ALMA view on V838 Mon

Schilke, P., Walmsley, C. M., Pineau des Forets, G., \& Flower, D. R. 1997, A\&A, 321,293

Schneider, F. R. N., Ohlmann, S. T., Podsiadlowski, P., et al. 2020, MNRAS, 495, 2796

Smette, A., Sana, H., Noll, S., et al. 2015, A\&A, 576, A77

Soker, N., \& Tylenda, R. 2007, MNRAS, 375, 909

Sparks, W. B., Bond, H. E., Cracraft, M., et al. 2008, AJ, 135, 605

Steffen, W., Koning, N., Wenger, S., Morisset, C., \& Magnor, M. 2011, IEEE Trans. Vis. Comput. Graph., 17, 454

Tokovinin, A. 2020, MNRAS, 496, 987

Tyas, L. M. G. 2012, PhD thesis, Durham University, UK

Tylenda, R. 2004, A\&A, 414, 223

Tylenda, R. 2005, A\&A, 436, 1009

Tylenda, R., \& Kamiński, T. 2012, A\&A, 548, A23
Tylenda, R., \& Soker, N. 2006, A\&A, 451, 223

Tylenda, R., Soker, N., \& Szczerba, R. 2005, A\&A, 441, 1099

Tylenda, R., Kamiński, T., \& Schmidt, M. 2009, A\&A, 503, 899

Tylenda, R., Hajduk, M., Kamiński, T., et al. 2011a, A\&A, 528, A114

Tylenda, R., Kamiński, T., Schmidt, M., Kurtev, R., \& Tomov, T. 2011b, A\&A, 532, A 138

Usov, V. V. 1991, MNRAS, 252, 49

Vernet, J., Dekker, H., D’Odorico, S., et al. 2011, A\&A, 536, A105

Wagner, R. M., \& Starrfield, S. G. 2002, IAU Circ., 7992, 2

Wisniewski, J. P., Morrison, N. D., Bjorkman, K. S., et al. 2003, ApJ, 588, 486

Wisniewski, J. P., Clampin, M., Bjorkman, K. S., \& Barry, R. K. 2008, ApJ, 683, L171

Wright, E. L., Eisenhardt, P. R. M., Mainzer, A. K., et al. 2010, AJ, 140, 1868 


\section{Appendix A: Optical spectra}

We used the X-shooter spectrograph on the VLT to obtain spectra of V838 Mon. The observations were executed on 6 January 2012 in nodding mode as program 088.D-0112(A). The seeing condition varied over the project execution between 0.57 and 1 1. 85 . Slit widths of $1.3,0.7$, and 0.4 arcsec were used for UV, VIS, and NIR arms of the spectrograph (Vernet et al. 2011), respectively. This resulted in a resolution of about 11500 in the VIS and NIR arms, and of 4100 in the NIR part. Additionally, spectra with a wide slit of $5^{\prime \prime}$ were secured for a better absolute flux calibration. The spectra cover the range $0.3-2.4 \mu \mathrm{m}$. All spectra were reduced with the most recent version of the esorex pipeline (source kit 3.5.0 from May 2020) in the ESO Reflex environment. Three exposures in each arm were taken and combined. Spectra were corrected for telluric absorption using the standalone Molecfit program (Kausch et al. 2015; Smette et al. 2015). The correction was calibrated on carefully selected bands in the spectra of the science object, avoiding water bands intrinsic to the star. A correction to the Solar System barycenter was applied and spectra were corrected for the interstellar extinction with $E_{B-V}$ of $0.85 \mathrm{mag}$ and $R_{V}=3.1$.

An optical spectrum in the range $370-890 \mathrm{~nm}$ was acquired at the South African Large Telescope (SALT) telescope under project number 2020-2-DDT-001. The observations were taken on 23 December 2020 with seeing of 1". 5 . We used the High Resolution Spectrograph (HRS Tyas 2012) in its high-resolution mode, that is, with $R \approx 40000$. HRS is a dual-beam fiber-fed spectrograph. We used a fiber entrance diameter of 1 '.56. Data were automatically reduced with the default instrument pipeline ${ }^{4}$ (Crawford et al. 2010). Spectra from five exposures were averaged. Shortward of $4500 \AA$, the spectrum is underexposed with a signal-to-noise ratio of a few. The SALT spectra are not calibrated in absolute flux units. No correction for telluric spectrum could be applied. We used spectra corrected to the Solar System barycenter.

The optical spectra represent sky regions of roughly the size of the respective seeing FWHM given above. In all cases, the regions encompass both stars and are larger than the entire remnant mapped with ALMA.

\section{Appendix B: SED data sources}

The data used in our SED reconstruction are listed in Table B.1. The $U B V R_{C} I_{C}$ photometry was taken from Goranskij et al. (2020) through the website of V. Goranskij ${ }^{5}$. We averaged measurements within about $0.5 \mathrm{yr}$ from the epoch of ALMA observations. Three standard deviations related to source variability in that period added to the photometric uncertainties given in Goranskij et al. (2020) serve as our effective measurement error. The broadband magnitudes were transformed to flux units using generic zero points of the Johnson and Cousins photometric systems for Vega.

Near-infrared photometric observations were carried out on UT 16.72 February 2019 at the $1.2 \mathrm{~m}$ telescope of the Mount Abu Infrared Observatory in India and using the Near-Infrared Camera/Spectrometer (NICS) equipped with a $1024 \times 1024 \mathrm{HgCdTe}$ Hawaii 1 array. The camera has an unvignetted $8^{\prime} \times 8^{\prime}$ field of view and uses $J H K_{s}$ filters that conform to Maunakea Observatories (MKO) specifications. Frames in each filter were obtained in five dithered positions, typically offset by $40^{\prime \prime}$, and with multiple frames (typically five) obtained in each dithered position.

\footnotetext{
4 http://pysalt.salt.ac.za/

5 http://www.vgoranskij.net/v838mon.ne3
}

The total exposure time in the individual filter was $8 \mathrm{~s}$. Standard procedures of dark and flat field corrections were applied. The corrected science frames were added to produce an average frame on which photometry was performed. Aperture photometry was derived using routines in IRAF, with the 2MASS field stars used for photometric calibration. The measured magnitudes $J=6.89( \pm 0.03), H=5.86( \pm 0.03)$, and $K=5.08( \pm 0.06)$ were converted to flux units using 2 MASS zero points.

We extracted data observed with the Wide-field Infrared Survey Explorer (WISE) using the WISE point source catalog (Wright et al. 2010). They represent the full period of WISE observations of V838 Mon, that is, from April to October 2010. Catalog magnitudes were converted to fluxes using standard zero points and with no color corrections. Uncertainties have a meaning of $3 \sigma$.

On UT 29 January 2018, V838 Mon was observed with COMICS (Kataza et al. 2000; Okamoto et al. 2003) on the Subaru $8.2 \mathrm{~m}$ telescope in imaging mode with the N12.4 filter $(12.4 \mu \mathrm{m}$ central wavelength and a width of $1.2 \mu \mathrm{m})$. COMICS in imaging mode feeds a $320 \times 240$ pixel $\mathrm{Si}$ :As array whose 0 ' 13 pixel $^{-1}$ plate scale affords a field of view of $41^{\prime \prime} .6 \times$ 31.2'. . Observations were chopped only (keeping all beams on chip) to maximize efficiency. The chopping throw used was $10^{\prime \prime}$ along a position angle of $\approx 0^{\circ}$. Chop pairs are differenced to remove the rapidly fluctuating background signal, then combined to yield the final reduced image. The flux calibration standard star HR 2639 (K5 III) was observed immediately before V838 Mon, and the adopted flux density for it in the $N 12.4$ band was determined from the WISE $W 3$ measurement scaled to $12.4 \mu \mathrm{m}$.

Far-infrared imaging photometry was obtained for V838 Mon on UT 24 October 2017 with HAWK+ (Harper et al. 2018) on board the Stratospheric Observatory for Infrared Astronomy (SOFIA). Images were obtained in total intensity mode in Bands $\mathrm{A}$ and $\mathrm{C}$ with Lissajous scan imaging. Data were reduced with $\mathrm{CRUSH}$ within the HAWC+ facility data reduction pipeline version 1.3.0. Level 3 pipeline-reduced and calibrated data products are obtained from the SOFIA science archive. Aperture photometry is performed directly on the flux-calibrated images with an aperture size sufficient to encircle all flux from V838 Mon (for Band C the source image appears partially elongated along one direction, most likely due to an instrument or aircraft artifact); it is not clear if an aperture correction is necessary to compensate. Flux uncertainties are estimated from the standard deviation of ten photometric extractions with the same aperture made at random positions in the blank field around V838 Mon.

For reference, we add to the SED in Fig. 11 MIR and FIR measurements from 2007. These are data from Spitzer and Gemini telescopes. They were reported by Wisniewski et al. (2008). For the comparison with the simulated SED, all data were corrected for interstellar extinction with $E_{B-V}=0.85 \mathrm{mag}$ and $R_{V}=3.1$, following Tylenda et al. (2005).

\section{Appendix C: Channel maps}

Figure C.1 presents maps of $\mathrm{SiO}$ emission at selected velocities. These maps were used to construct the shape of the $\mathrm{SiO}$ region shown in Fig. 12 and discussed in Sect. 6.2. For comparison, in Fig. C. 2 we present channel maps for CO emission. 


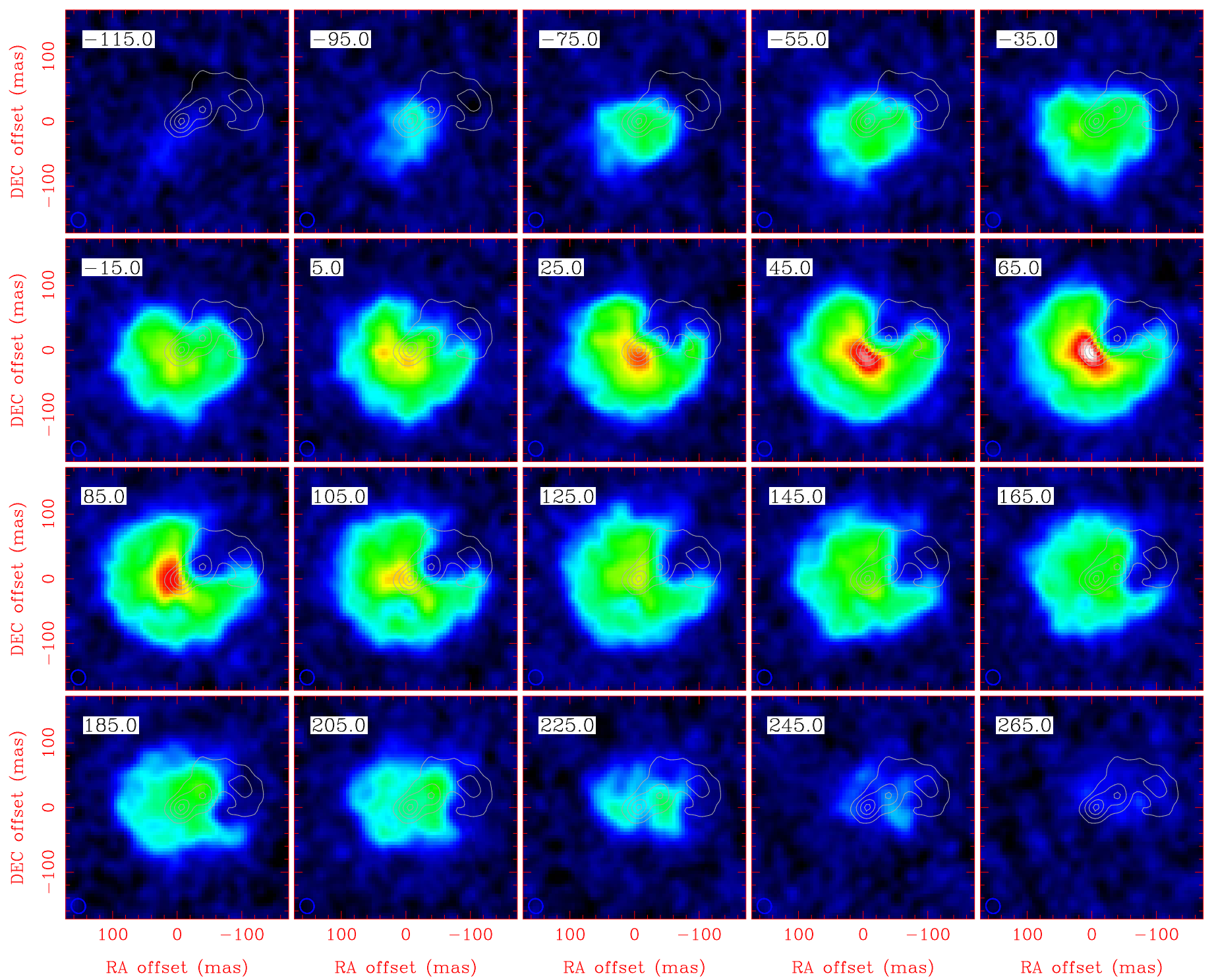

Fig. C.1. Channel maps of $\mathrm{SiO}$ emission at Briggs weighting with the robust parameter of 0.5 and a beam size of 30 mas. Contours show continuum emission at $10,30,60$, and $90 \%$ of the peak emission. Velocity is indicated in each map in $\mathrm{km} \mathrm{s}^{-1}$. Colorscale is linear from -0.4 to $7.0 \mathrm{mJy}$ per beam.

\section{Appendix D: Effects of radiation pressure of the B star on the ejecta}

When approaching the B3V star, the flow from V838 Mon is affected by the gravity of the star and the radiation pressure. The effective acceleration, $a_{\mathrm{eff}}$, of the matter at a distance $r$ from the star of a mass $M_{s}$ and a luminosity $L_{s}$ is thus

$$
a_{\mathrm{eff}}=g+a_{\text {rad }}
$$

where

$$
g=-G M_{s} / r^{2}
$$

and

$$
a_{\text {rad }}=\left(L_{s} /\left(4 \pi r^{2} c\right)\right)\left(\pi a^{2} Q_{p r}\right) /\left((4 / 3) \pi a^{3} \rho_{d} / R_{d g}\right)
$$

Here, $a$ is the dust grain radius, $Q_{p r}$ is the radiation pressure efficiency factor, $\rho_{d}$ is the density of the grain material, $R_{d g}$ denotes the dust-to-gas mass ratio, and $G$ is the gravitational constant.

Both $g$ and $a_{\text {rad }}$ have the same dependence on the distance from the star $\left(r^{-2}\right)$, and so their ratio is independent of the distance. Therefore, it is convenient to rewrite the above formula as

$a_{\mathrm{eff}}=g\left(1+a_{\text {rad }} / g\right)=g\left(1-(3 / 16 \pi) L_{s} /\left(G M_{s} c\right)\left(Q_{p r} R_{d g} /\left(a \rho_{d}\right)\right)\right.$.

Substituting values of the physical constants and expressing the stellar luminosity, $l_{s}$, and mass, $m_{s}$, in solar units, one obtains

$$
a_{\mathrm{eff}}=g\left(1-5.7410^{-5}\left(l_{s} / m_{s}\right)\left(Q_{p r} R_{d g} /\left(a \rho_{d}\right)\right) .\right.
$$

We adopt typical parameters of a B3 V star, i.e., $m_{s}=8.0, l_{s}=$ 1900 , as well as $\mathrm{T}_{\text {eff }} \simeq 18000 \mathrm{~K}$ and $\mathrm{R}_{s} \simeq 5.0 \mathrm{R}_{s}$. We also adopt "astronomical silicate" dust with $\rho_{d} \simeq 3.0 \mathrm{~g} \mathrm{~cm}^{-3}, R_{d g}=0.01$, and $a=0.1 \mu \mathrm{m}$. For the above effective temperature and grain radius, $Q_{p r} \simeq 1.4$ (from B. Draine's web page ${ }^{6}$ ). Substituting these values yields

$$
a_{\mathrm{eff}} \simeq g(1-6.4)=-5.4 g .
$$

Thus, in the absence of other effects, the flow around the B3 V companion is dominated by the stellar radiation pressure on the

6 https://www . astro.princeton. edu/ draine/dust/dust . diel.html 


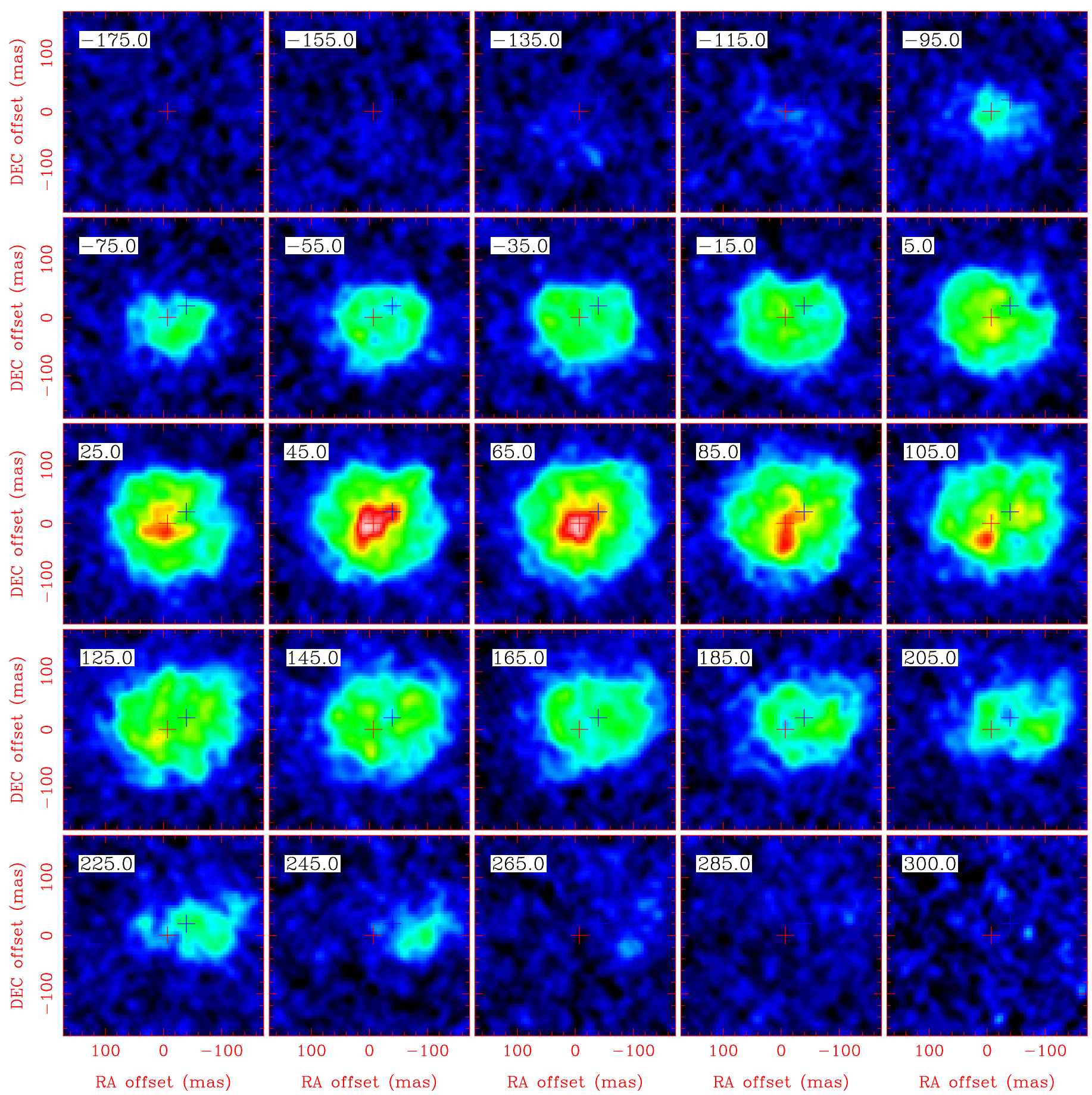

Fig. C.2. Channel maps of $\mathrm{CO}$ emission at Briggs weighting with the robust parameter of 0.5 and a beam size of 28 mas. The red and blue crosses mark the locations of the $\mathrm{M}$ and $\mathrm{B}$ components, respectively. The range of velocities covered here is wider than for SiO channel maps in Fig. C.1. Colorscale is linear from -0.4 to $4.0 \mathrm{mJy}$ per beam.

dust grains rather than the stellar gravitational field. To illustrate the expected effects on the flow in the vicinity of the B star, we integrated the standard (ballistic) equation of motion for particles ejected by V838 Mon with a given velocity in directions towards the companion. The acceleration in the above form is the only factor governing the motion of the particles. No (hydrodynamic) effects of possible crossing of the particle trajectories were taken into account. Figure D.1 shows the case of pure gravity $\left(a_{\text {rad }}=0\right)$. V838Mon is at the coordinate origin $(x=0$ and $y=0$ ), B $3 \mathrm{~V}$ star is at $x=0$ and $y=250$ au (shown by an asterisk in the figure). Particles are injected at $(0,0)$ with a velocity of $200 \mathrm{~km} \mathrm{~s}^{-1}$ and at different (small) angles from the $y$ axis (i.e., a line joining V838 Mon and B3). Due to the gravity of the companion, the trajectories are bent toward the axis of symmetry $(x=0)$ and those passing close to the star intersect behind it. In reality, we expect that a turbulent wake is produced in the flow behind the perturbing star.

Figure D. 2 presents the case when $a_{\text {eff }}=-5 g$. Other parameters remain the same as above. The radiation pressure slows down and rejects the particles approaching the star too closely. This creates an empty cone in the immediate vicinity of and behind the B star. The effect is stronger when the flow is slower. 
Table B.1. Flux measurements of V838 Mon.

\begin{tabular}{ccccc}
\hline Filter & $\begin{array}{c}\lambda_{\text {eff }} \\
(\mu \mathrm{m})\end{array}$ & $\begin{array}{c}\text { Flux } \\
(\mathrm{Jy})\end{array}$ & $\begin{array}{c}\text { Relative } \\
3 \sigma \text { error }\end{array}$ & Ref. \\
\hline$U$ & 0.37 & $0.07 \mathrm{e}-3$ & 0.012 & 1 \\
$B$ & 0.44 & $1.80 \mathrm{e}-3$ & 0.014 & 1 \\
$V$ & 0.55 & $1.45 \mathrm{e}-2$ & 0.023 & 1 \\
$R_{C}$ & 0.65 & $9.06 \mathrm{e}-2$ & 0.009 & 1 \\
$I_{C}$ & 0.79 & 0.5441 & 0.012 & 1 \\
$J$ & 1.25 & 2.796 & 0.003 & 2 \\
$H$ & 1.64 & 4.638 & 0.006 & 2 \\
$K_{S}$ & 2.20 & 6.193 & 0.015 & 2 \\
$W 1$ & 3.35 & 4.17 & 0.12 & 3 \\
$W 2$ & 4.60 & 5.63 & 0.11 & 3 \\
$W 3$ & 11.56 & 35.83 & 0.19 & 3 \\
$N 12.4$ & 12.40 & 16.1 & 0.15 & 2 \\
$W 4$ & 22.09 & 40.421 & 0.005 & 3 \\
HAWK+ A & 53.09 & 11.5 & 0.31 & 2 \\
HAWK+ C & 88.67 & 4.1 & 0.37 & 2 \\
ALMA B6 & 1333.87 & $2.3 \mathrm{e}-3$ & 0.01 & 2 \\
\hline
\end{tabular}

References. (1) Goranskij et al. (2020); (2) this study; (3) Wright et al. (2010).

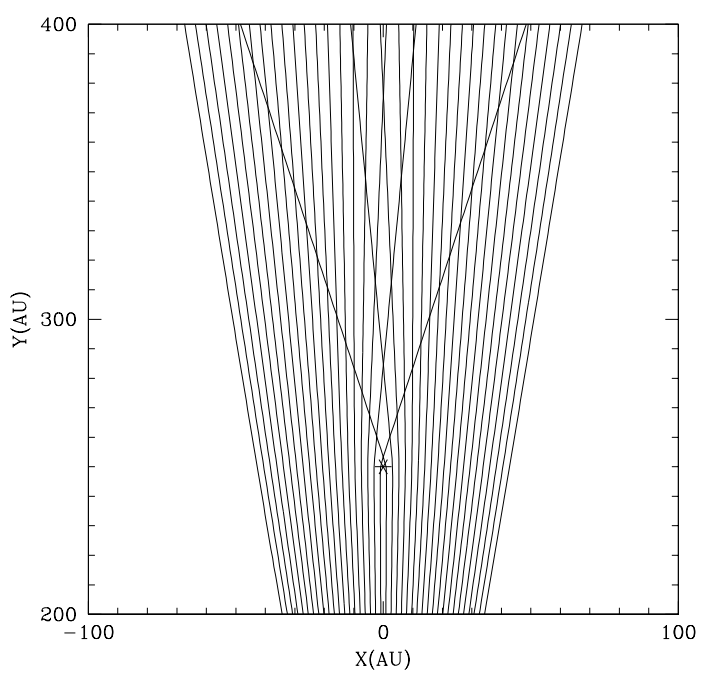

Fig. D.1. Particle trajectories $\left(v_{0}=200 \mathrm{~km} \mathrm{~s}^{-1}\right)$ in the vicinity of the $\mathrm{B} 3 \mathrm{~V}$ companion in the case of $a_{\mathrm{eff}}=g$ (pure gravity).

The trajectories intersect, especially in the vicinity of the star. In reality, this should result in an increase of the density of the flow and disordered (turbulent) motions, especially near the edge of the cone. Since the velocities involved are highly supersonic, a creation of shock fronts is also likely.

The above considerations do not take into account dust sublimation that is expected to take place close to the star. If dust is destroyed at a sublimation temperature, $T_{d}$, then it takes place at a distance, $r_{d}$, which can be estimated from

$$
\left(4 \pi R_{s}^{2} \sigma T_{s}^{4}\right) /\left(4 \pi r_{d}^{2}\right) \pi a^{2} Q_{\mathrm{abs}}\left(T_{s}\right)=4 \pi a^{2} \sigma T_{d}^{4} Q_{\mathrm{em}}\left(T_{d}\right) .
$$

Here $R_{s}$ is the radius of the star whereas $Q_{\mathrm{abs}}$ and $Q_{\mathrm{em}}$ are the Planck-averaged dust absorption and emission coefficients, respectively. The above equation can be rewritten as

$$
\left(R_{s} / r_{d}\right)^{2}=4 Q_{\mathrm{em}} / Q_{\mathrm{abs}}\left(T_{d} / T_{s}\right)^{4}
$$

or

$$
r_{d} / R_{s}=\left(Q_{\mathrm{abs}} / 4 Q_{\mathrm{em}}\right)^{1 / 2}\left(T_{s} / T_{d}\right)^{2} .
$$

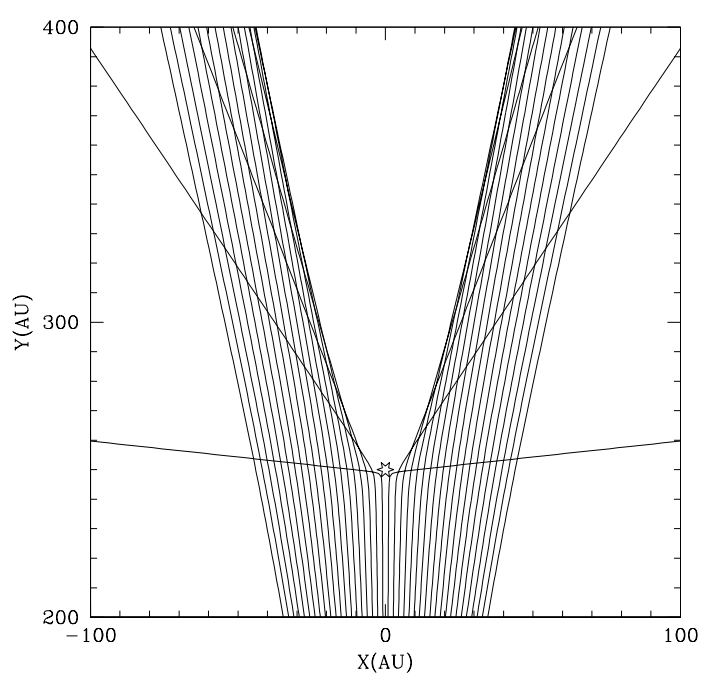

Fig. D.2. Same as in Fig. D.1 but for $a_{\text {eff }}=-5 g$.

Assuming that the silicate grains sublimate at $T_{d} \simeq 1350 \mathrm{~K}$ (Lenzuni et al. 1995) and taking the respective $Q$ values from the website of B. Draine $\left(Q_{\mathrm{abs}}(18000 \mathrm{~K}) / Q_{\mathrm{em}}(1350 \mathrm{~K}) \simeq 31.3\right)$, we finally obtain

$$
r_{d} \simeq 500 R_{s}=11 \mathrm{au} .
$$

(In our 3D model introduced in Sect. 6.1, which was constructed with similar grains and the same stellar parameters, the clearing around the B star is $10 \mathrm{au}$, consistent with the calculation here.) We next assume that if the matter is able to penetrate inside $r_{d}$ then there is no dust and consequently $a_{\text {rad }}=0$ and $a_{\text {eff }}=g$. In this approach, the sublimation in instantaneous. We further assume that dust is not formed again when the matter leaves the region where $r<r_{d}$. Figure D.3 shows the results of including the sublimation effect. Particles ejected with larger angles from the $x$ axis do not approach the star beyond $r_{d}$, so their trajectories are the same as in Fig. D.2. On the other hand, particles ejected at small angles are able to penetrate further than $r_{d}$ and their trajectories are similar (but not the same) as in Fig. D.2. Going back to the case of the V838 Mon ejecta and its companion, we can expect that with dust sublimation, the B $3 \mathrm{~V}$ companion can form both an extended cone of compressed matter and a turbulent wake.

The results are sensitive to the velocity of the flow. For instance, for $v_{0} \simeq 85 \mathrm{~km} \mathrm{~s}^{-1}$, the matter is nowhere able to penetrate inside $r_{d}$ and no wake is formed, as illustrated in Fig. D.4.

\section{Appendix E: Shocks induced by the wind of the B star}

As noted above, the stellar radiation pressure potential has the same dependence on the distance from the star as the gravitational potential (with the opposite sign). Therefore, by analogy to the formula for the free-fall velocity in the gravitational field, the stopping radius, $r_{\text {stop }}$, at which the inflowing dusty matter is stopped in the stellar radiation field, can be derived from

$$
v_{0}=\left(2 f G M_{s} / r_{\text {stop }}\right)^{1 / 2}
$$

where $f=a_{\mathrm{eff}} / g$. Thus, 


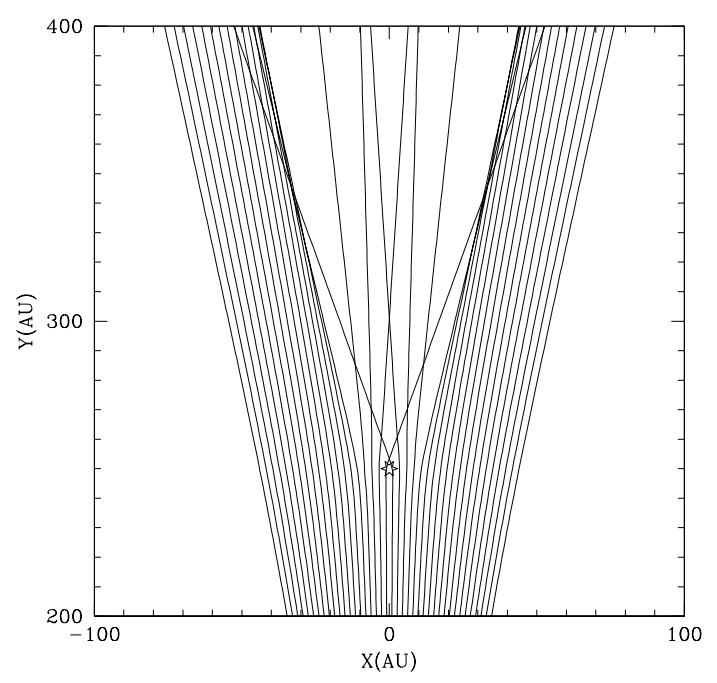

Fig. D.3. Same as in Fig. D.2 but with dust evaporation at $r<r_{d}$.

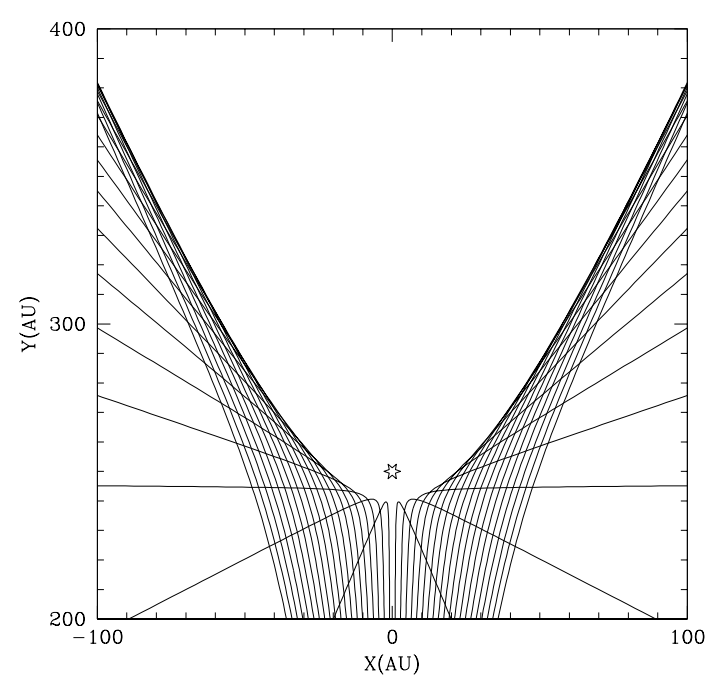

Fig. D.4. Same as Fig. D. 3 but for $v_{0}=85 \mathrm{~km} \mathrm{~s}^{-1}$.

The distance from the star, $r_{\mathrm{sh}}$, where the stellar wind stops and shocks the inflowing matter can be estimated from a standard condition of momentum flux equilibrium, that is

$$
N_{0} v_{0}^{2}=N_{w} v_{w}^{2},
$$

where the subscript " 0 " refers to the flow parameters, while " $w$ " - to those of the stellar wind. Thus,

$$
N_{0} v_{0}^{2}=v_{w} \dot{M}_{w} /\left(4 \pi r_{\mathrm{sh}}^{2} m_{p}\right)
$$

where $\dot{M}_{w}$ is the wind mass-loss rate and $m_{p}$ denotes the particle mass. Finally,

$$
r_{\mathrm{sh}}=\left(\dot{M}_{w} v_{w} /\left(4 \pi m_{p} N_{0} v_{0}^{2}\right)\right)^{1 / 2} .
$$

Let us apply the above formulae to the parameters expected for the $\mathrm{B} 3 \mathrm{~V}$ companion and the observed outflow from V838Mon in the vicinity of the companion, with $v_{0}=75$ $250 \mathrm{~km} \mathrm{~s}^{-1}, N_{0}=10^{6-7} \mathrm{~cm}^{-3}, \dot{M}_{w}=(1-4) \times 10^{-11} M_{\odot} \mathrm{yr}^{-1}$, and $v_{w}=800-1400 \mathrm{~km} \mathrm{~s}^{-1}$. The calculated radii are given in Table E.1 and can be summarized as follows. About $5 \mathrm{yr}$ after the
Table E.1. Radii where ejecta is stopped and shocked by the wind of the B star.

\begin{tabular}{cccrccc}
\hline $\begin{array}{c}v_{0} \\
\mathrm{~km} \mathrm{~s}^{-1}\end{array}$ & $\begin{array}{c}N_{0} \\
\mathrm{~cm}^{-3}\end{array}$ & $\begin{array}{c}\dot{M}_{w} \\
\mathrm{M}_{\odot} \mathrm{yr}^{-1}\end{array}$ & $\begin{array}{c}v_{w} \\
\mathrm{~km} \mathrm{~s}^{-1}\end{array}$ & $\begin{array}{c}r_{\text {stop }} \\
\mathrm{au}\end{array}$ & $\begin{array}{c}r_{\mathrm{sh}} \\
\mathrm{au}\end{array}$ & $\begin{array}{c}r_{d} \\
\mathrm{au}\end{array}$ \\
\hline 250 & $10^{7}$ & $1 \cdot 10^{-11}$ & 800 & 1.2 & 0.04 & 10.4 \\
250 & $10^{7}$ & $4 \cdot 10^{-11}$ & 1400 & 1.2 & 0.11 & 10.4 \\
75 & $10^{6}$ & $1 \cdot 10^{-11}$ & 800 & 13.7 & 0.44 & 10.4 \\
75 & $10^{6}$ & $4 \cdot 10^{-11}$ & 1400 & 13.7 & 1.15 & 10.4 \\
\hline
\end{tabular}

Notes. For comparison, $r(\mathrm{~B} 3 \mathrm{~V})=5.0 \mathrm{R}_{\odot}=0.02 \mathrm{au}$.

V838 Mon eruption, the fastest and probably the densest ejecta reached the B3 V companion. Dust was evaporated well before the matter could have been stopped by radiation pressure on dust grains $\left(r_{d} \gg r_{\text {stop }}\right)$. As a result, the matter could have approached the companion until it had been shocked and stopped by the stellar wind a few stellar radii above the stellar surface. Probably no matter was then accreted on the companion. Instead, a hot shocked region was then formed above the photosphere.

At the ALMA epoch and at present, the matter that flows in the vicinity of the companion is much slower and probably less dense. Therefore, it is stopped by the radiation pressure about 1000 stellar radii above the stellar photosphere, well before the dust grains can evaporate. No matter is accreted on the companion. The wind collides with the inflowing matter at $r_{\text {stop }}$ but its dynamical influence is negligible. A rarefied hot region is probably formed near $r_{\text {stop }}$. 